\title{
Are elevated moist layers a blind spot for hyperspectral infrared sounders? - A model study
}

\author{
Marc Prange ${ }^{1}$, Manfred Brath ${ }^{1}$, and Stefan A. Buehler ${ }^{1}$ \\ ${ }^{1}$ Universität Hamburg, Meteorologisches Institut, Bundesstraße 55, 22525 Hamburg, Germany \\ Correspondence: Marc Prange (marc.prange@uni-hamburg.de)
}

\begin{abstract}
The ability of the hyperspectral satellite based passive infrared instrument IASI to resolve Elevated Moist Layers (EMLs) within the free troposphere is investigated. EMLs are strong moisture anomalies with significant impact on the radiative heating rate profile and are thought to be coupled to freezing level detrainment of convective cells in the tropics. Based on an exemplary EML testcase and forward modelled IASI observations, it is shown that if sufficient independent humidity and temperature information is available, EMLs do not pose a blind spot for passive satellite observations, contrary to what results of Stevens et al. (2017) have indicated. To further quantify the retrieval's ability to capture moisture anomalies, a statistical evaluation of synthetic retrievals of 1438 clear sky tropical ocean short-range forecast model atmospheres is conducted. For this purpose, a framework for the identification and characterisation of moisture anomalies, a subset of which are EMLs, is introduced. The statistical evaluation shows that retrieved moisture anomalies are on average $37 \%$ weaker and $28 \%$ thicker than their true counterparts, which can be attributed to the retrieval smoothing error and the fact that rather weak and narrow moisture anomalies are most frequently missed by the retrieval. Smoothing is found to also constrain the magnitude of local heating rate extremes associated with moisture anomalies, particularly for the strongest anomalies that are found in the lower to mid troposphere. In total, about $72 \%$ of moisture anomalies in the reference dataset are found by the retrieval. Below $5 \mathrm{~km}$ altitude, this fraction is only on the order of $30 \%$, which can be attributed to the fact that lower tropospheric moisture anomalies are typically more narrow and therefore tougher to retrieve than anomalies aloft. We conclude that the retrieval of lower to mid tropospheric moisture anomalies, in particular of EMLs, is possible when the anomaly is sufficiently strong and its thickness is at least on the order of about $1.5 \mathrm{~km}$. This study sets the methodological basis, from a retrieval setup and evaluation perspective, to investigate real world EMLs in IASI observations in the future.
\end{abstract}

\section{Introduction}

The vertical structure of tropospheric water vapor is an important driver for dynamical processes due to its effect on the radiative heating profile. In particular, Muller and Bony (2015) found that the spatial variability of the radiative heating profile gives rise to spatial self-aggregation of convection, which is thought to be a key factor for uncertainties in climate projections (Bony et al., 2015; Mauritsen and Stevens, 2015). A contributing phenomenon to the spatial variability in radiative heating profiles are moisture inversions in the tropical lower to mid free troposphere, so called Elevated Moist Layers (EMLs). To our best knowledge, EMLs were first identified by Haraguchi (1968) over the tropical eastern Pacific and independently by 
Ananthakrishnan and Kesavamurthy (1972) over India. A first systematic connection of these EMLs to the freezing level was brought to attention by Johnson et al. (1996), who formally distinguished between the commonly referred to trade wind inversion between 2 and $3 \mathrm{~km}$ (Cao et al., 2007) and another stable layer aloft that manifests during summer months just below the freezing level. Both, the trade wind inversion and the stable layer at the freezing level are capable of trapping moisture beneath and forming strong vertical humidity gradients. The stable layer around the freezing level has recently been brought to attention again within the framework for assessing the tropical lower tropospheric moisture budget introduced by Stevens et al. (2017).

While the general role of EMLs within their meso-scale environment has not yet been assessed conclusively, there are conceptual ideas about the emergence of EMLs and their impact on meso-scale atmospheric dynamics. Johnson et al. (1996) and Stevens et al. (2017) both hypothesise that EMLs preferably emerge in the vicinity of moist convective cells that penetrate the freezing level, where enhanced stability leads to detrainment of the saturated air. Stevens et al. (2017) further highlight the stabilising effect of glaciation above the freezing level within the initial convective cell on the environment, which further impedes nearby convection from penetrating the freezing level, leading to increased cloudiness and moisture. Studies investigating vertical modes of cloudiness in the tropics further support the idea of preferred convective detrainment near the freezing level (Zuidema, 1998; Johnson et al., 1999; Posselt et al., 2008). Following the findings of Muller and Bony (2015), EMLs may also contribute to the maintenance and aggregation of convection via the strong vertical gradient they induce in the radiative heating profile. The strong cooling at the EML top induces subsidence and horizontal mass convergence, while near the surface a mass divergence is induced. The mass divergence near the surface in the vicinity of convection may act to maintain the convection.

Stevens et al. (2017) conducted an observational case study of an EML present during the NARVAL-2 (Next Generation Remote Sensing for Validation Studies) measurement campaign. One method they deployed was a satellite retrieval analysis based on passive microwave and infrared observations, both of which showed very poor performance in capturing the EML structure, suggesting that EMLs present a somewhat fundamental blind spot for passive satellite observations. This study aims at more comprehensively investigating the question whether EMLs really present a blind spot and where the limitations in resolving such moisture structures are, based on the IASI (Infrared Atmospheric Sounding Interferometer) instrument. We start out with the hypothesis that due to the EMLs association with particularly stratified layers, a good representation of the vertical temperature structure is key for resolving the moisture structure.

Instead of revolving around real satellite observations, the framework of this study is based on model atmospheres that are fed into a radiative transfer model to obtain synthetic satellite observations, which are then used to conduct the retrieval. This methodology has the advantage that it allows for a very controlled environment, where undesired sources of error can simply be excluded, for example collocation errors or instrumental issues. This allows for a clearer attribution of retrieval errors in the end and therefore a clearer understanding of the retrievability of EMLs.

This study is structured as follows. In Sect. 2 the retrieval setup is introduced, including a description of the retrieval algorithm, the representation of the IASI instrument by the radiative transfer model and the a priori assumptions. Section 3 introduces a framework for identifying and characterising moisture anomalies, which is extensively used later on to specifically 
assess the retrieval's ability to capture moisture anomalies. In Sect. 4 a case study is conducted, where the introduced retrieval is applied to an EML scenario that is similar to the one investigated by Stevens et al. (2017). Besides the introduced retrieval setup, another setup that does not retrieve the temperature profile is applied to investigate the importance of capturing the temperature structure for the humidity retrieval. Then the retrieval is applied to an ensemble of 1438 clear-sky atmospheric profiles over the tropical ocean, which are part of the ECMWF diverse profile database introduced by Eresmaa and McNally (2014). Based on that, the absolute retrieval error and the smoothing error are quantified statistically in Sect. 5. Based on the framework introduced in Sect. 3 for identifying and characterising moisture anomalies, the retrieval's ability to capture the moisture structures of the test dataset and their footprint on the heating rate profile is assessed in Sect. 6 . The results are summarized and final conclusions are drawn in Sect. 7.

\section{The retrieval}

Extracting atmospheric state variables such as the temperature or concentrations of atmospheric constituents from passive satellite observations generally poses an inversion problem. The forward problem of calculating spectral radiances for a given atmospheric state in the clear sky case can be solved by a radiative transfer model (also "forward model") because the physical concepts of clear sky radiative transfer are well understood and quantified. However, due to the ill-posedness of the inverse problem and the non-linear nature of the forward model, a retrieval requires sophisticated methods to regularise the problem and find an optimal solution.

For the purpose of passive remote sensing of the vertical structure of clear-sky atmospheric constitution and temperature, the so called Optimal Estimation Method (OEM) is an established approach to solve the inverse problem. OEM is an inversion approach based on Bayes Theorem, where a priori assumptions and the measurement are represented by Gaussian probability distributions that are combined to obtain an a posteriori conditional probability distribution, the expected value of which is taken as the solution. Typically, iterative schemes that minimise a cost function are used to account for the forward model non-linearity. The formalism used in this work strongly follows the comprehensive framework introduced by Rodgers (2000). Within the next subsections the technical implementation of the retrieval setup used in this study is introduced.

\subsection{Spectral setup}

The retrieval setup of this study aims at resolving the vertical structure of water vapor in the troposphere, with particular focus on EML scenarios. An important absorption band of water vapor in the thermal infrared is the rotational-vibrational band centered around $6.25 \mu \mathrm{m}\left(1594.78 \mathrm{~cm}^{-1}\right.$ ) (see Fig. 1), which offers rich vertically distributed information. The exact frequency selection is based on the study of Schneider and Hase (2011), who demonstrated the suitability of the spectral region between 1190 to $1400 \mathrm{~cm}^{-1}$ for retrieving profiles of water vapor and its main isotopologues.

The spectral signal of water vapor depends not only on the atmospheric water vapor itself but also on the temperature. Therefore, unresolved temperature features may falsely be interpreted as water vapor signals. We assume that this is particularly relevant for EML scenarios because the strong vertical humidity gradients typically go along with temperature inversions. If 
these temperature inversions are unresolved, their signal interferes with the retrieval's ability of correctly capturing the EML structure. Schneider and Hase (2011) and Borger et al. (2018) concurrently found that temperature induced errors can yield up to $15 \%$ relative error for the lower to mid tropospheric $\mathrm{H}_{2} \mathrm{O}$ retrieval, which is significant compared to other sources of error, such as interfering species. To reduce this error, we add independent temperature information to the retrieval from the spectral range between $2150-2400 \mathrm{~cm}^{-1}$, which is part of the $\mathrm{CO}_{2}$ absorption band centred around $4.8 \mu \mathrm{m}\left(2083 \mathrm{~cm}^{-1}\right)$. The blue shading in Fig. 1 in this spectral region indicates that there is almost no interference of water vapor absorption.

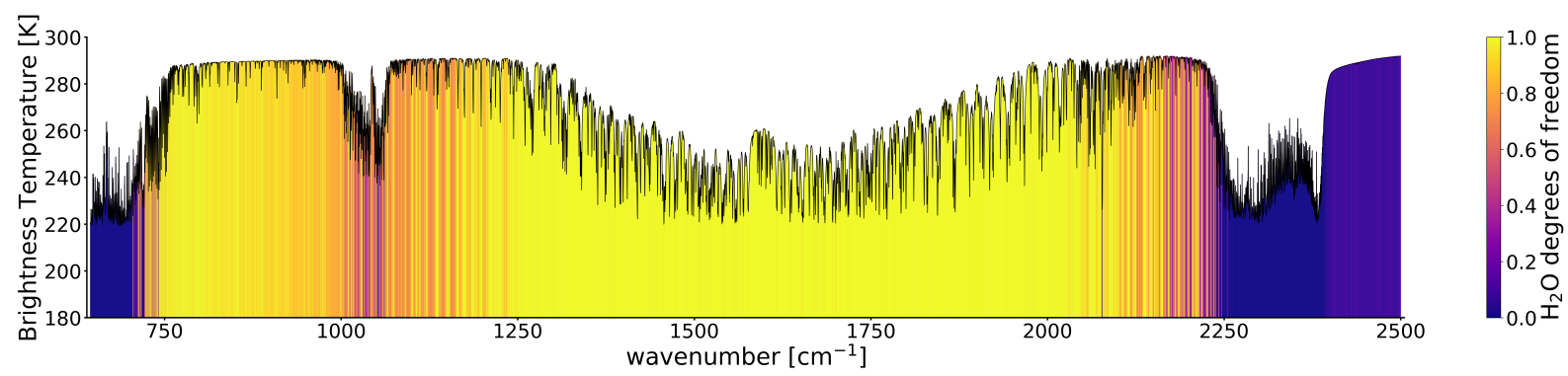

Figure 1. Forward simulated spectrum in the spectral range of the IASI instrument. Colors denote the water vapor information content at individual channels calculated as the trace of the averaging kernel matrix when only that channel is used.

To assure that the radiative background of the surface is represented well in the retrieval, 5 window channels are added to the spectral setup that have been identified by Boukachaba et al. (2015) as suited window channels. The channels are located at wavenumbers $901.5 \mathrm{~cm}^{-1}, 942.5 \mathrm{~cm}^{-1}, 943.25 \mathrm{~cm}^{-1}, 962.5 \mathrm{~cm}^{-1}$ and $1115.75 \mathrm{~cm}^{-1}$. The complete spectral setup encompasses 1845 channels.

\subsection{Retrieval quantities}

The quantities targeted for retrieval in this study are the profiles of water vapor volume mixing ratio $\left(\mathbf{V M R}_{\mathbf{H}_{2} \mathbf{O}}\right)$, temperature $(\boldsymbol{T})$ and the surface temperature $\left(T_{s}\right)$. They are represented by the retrieval state vector $\boldsymbol{x}$ :

$\boldsymbol{x}=\left(\begin{array}{c}\log \left(\mathbf{V M R}_{\mathbf{H}_{\mathbf{2}} \mathbf{O}}\right) \\ \boldsymbol{T} \\ T_{s}\end{array}\right)$

The water vapor profile is retrieved in natural logarithmic units, which is favourable for two reasons. On the one hand, $\mathrm{VMR}_{\mathrm{H}_{2} \mathrm{O}}$ is a quantity that ranges over several orders of magnitude from a few percent near the surface to $O\left(10^{-6}\right)$ in the upper troposphere and above, which is numerically inconvenient for the optimisation algorithm. On the other hand, the transformation to logarithmic units avoids the possibility of physically implausible negative VMR values.

The major interfering trace gas species in the chosen spectral region that are not part of the retrieval state vector $\boldsymbol{x}$ are $\mathrm{CH}_{4}$ and $\mathrm{N}_{2} \mathrm{O}$. Based on the error budget analysis conducted by Schneider and Hase (2011) it is not expected that these species 
are significant sources of error compared to errors in the temperature profile. Therefore, $\mathrm{CH}_{4}$ and $\mathrm{N}_{2} \mathrm{O}$ are excluded from the absorption setup in this study.

\subsection{Optimal estimation algorithm}

Formally, the inversion problem can be introduced by first considering the basic equation solved by the Forward Model F:

$\boldsymbol{y}=\mathbf{F}(\boldsymbol{x}, \boldsymbol{b})+\boldsymbol{\epsilon}$

with the retrieval state vector $\boldsymbol{x}$, an estimate of which is to be retrieved and the forward model parameters $\boldsymbol{b}$ that include profiles of other atmospheric absorption species, namely $\mathrm{N}_{2}, \mathrm{O}_{2}, \mathrm{CO}_{2}$ and Ozone. The Forward Model $\mathbf{F}$ calculates the spectral radiance along the assumed 1D path through the atmosphere and returns the spectrum $\boldsymbol{y}$, which represents the measurement of the sensor when neglecting measurement noise $\epsilon$. The inversion problem is to obtain $\hat{x}$, an estimate of the true $x$, from the observed spectrum $\boldsymbol{y}$. Based on Bayes Theorem, $\hat{\boldsymbol{x}}$ can be iteratively obtained using the Levenberg-Marquardt (LM) algorithm (Levenberg, 1944; Marquardt, 1963). The retrieved state $\hat{\boldsymbol{x}}$ is estimated iteratively after $i$ iterations by:

$\boldsymbol{x}_{i+1}=\boldsymbol{x}_{i}+\left[(1+\gamma) \mathbf{S}_{a}^{-1}+\mathbf{K}_{i}^{T} \mathbf{S}_{y}^{-1} \mathbf{K}_{i}\right]^{-1}\left[\mathbf{K}_{i}^{T} \mathbf{S}_{y}^{-1}\left[\boldsymbol{y}-\mathbf{F}\left(\boldsymbol{x}_{i}\right)\right]-\mathbf{S}_{a}^{-1}\left[\boldsymbol{x}_{i}-\boldsymbol{x}_{a}\right]\right]$

with the a priori covariance Matrix $\mathbf{S}_{a}$, the measurement covariance matrix $\mathbf{S}_{y}$ and the jacobian matrix $\mathbf{K}_{i}$ at iteration $i$. The first term on the right hand side of Eq. 3 is the current best estimate of the atmospheric state $\boldsymbol{x}$, which for $i=0$ will in this work assumed to be $\boldsymbol{x}_{0}=\boldsymbol{x}_{a}$. The second term on the right hand side of Eq. 3 can be viewed as being driven by the magnitude of $\mathbf{K}_{i}$ and by the difference between the measurement and the forward modelled spectrum $\left[\boldsymbol{y}-\mathbf{F}\left(\boldsymbol{x}_{\boldsymbol{i}}\right)\right]$. At the same time the difference $\left[\boldsymbol{x}_{\boldsymbol{i}}-\boldsymbol{x}_{\boldsymbol{a}}\right]$ constrains the magnitude of this term to avoid solutions too far off the a priori state. The covariances $\mathbf{S}_{a}$ and $\mathbf{S}_{y}$ act as weights to account for the uncertainty associated with the atmospheric state and the measurement.

The one variable not explained up to now is $\gamma$, which is a non-dimensional positive integer that allows the LM scheme to be a weighted version of both a Gauss-Newton scheme $(\gamma=0)$ and a gradient descent scheme $(\gamma=\infty)$. In our implementation, $\gamma=10$ is arbitrarily chosen as a starting value and is adjusted to assure a reduction of the cost function with every iteration. This is particularly advantageous for cases where the starting point is far away from the solution, in which the Gauss-Newton scheme may struggle to converge. This makes the LM scheme in general more robust than the Gauss-Newton scheme. The tradeoff is that for cases where the starting point is rather close to the solution the LM scheme typically converges slower than the Gauss-Newton scheme.

\subsection{The forward model and representation of IASI}

The radiative transfer model used in this study is version 2.3.1279 of the Atmospheric Radiative Transfer Simulator (ARTS). A comprehensive and compact description of ARTS is provided by Eriksson et al. (2011) and Buehler et al. (2018) and more 
documentation can be found on the ARTS website (https://www.radiativetransfer.org). Here, only the features that are directly relevant for the conducted retrieval calculations are presented.

For a body of a given temperature and emissivity ARTS calculates the emitted radiation and its transmission through a given atmospheric state on a line-by-line basis. Spectral line data were taken from the HITRAN (High Resolution Transmission) molecular spectroscopic database (Gordon et al., 2017) and continuum absorption of water vapor, oxygen, nitrogen and $\mathrm{CO}_{2}$ are represented by the MT_CKD model for continuum absorption (Mlawer et al., 2012).

The radiative transfer simulations are conducted as monochromatic pencil beams on a frequency grid with a resolution of $0.25 \mathrm{~cm}^{-1}$, which coincides with the spectral sampling interval of IASI. The obtained spectra are then convolved with a Gaussian weighting function with a Full Width at Half Maximum (FWHM) of $0.5 \mathrm{~cm}^{-1}$ to mimic the spectral response function of IASI. These technical specifications are taken from Coppens et al. (2019). To better represent the instrument, Gaussian noise with a standard deviation of $0.2 \mathrm{~K}$ for wavenumbers below $1750 \mathrm{~cm}^{-1}$ and $0.3 \mathrm{~K}$ above $1750 \mathrm{~cm}^{-1}$ is added to the forward simulated spectra (Clerbaux et al., 2009). The sensor is assumed to be in $850 \mathrm{~km}$ altitude and to have a nadir viewing direction. The atmospheric cases simulated are limited to clear-sky and are above ocean surfaces, where the surface emissivity in the spectral region covered by IASI is assumed to be 1 .

The ARTS internal OEM module, which is part of the latest release version 2.4.0 of ARTS, is used to conduct the actual retrieval calculations.

\subsection{A priori assumptions}

The a priori assumptions about the atmospheric state are defined as the knowledge about the state prior to the measurement. Although the true state is always known within the frame of this model study, the a priori knowledge is chosen based on information that would also be available in the situation of a real measurement. The a priori knowledge is represented by an a priori state vector $\boldsymbol{x}_{a}$ and a covariance matrix $\mathbf{S}_{a}$. For the definition of the a priori state the tropical mean atmospheric state from the profile database of Anderson et al. (1986) is used as a basis, which from now on will be referred to as tropical FASCOD (Fast Radiative Signature Code) atmosphere.

Where not stated otherwise, the a priori surface temperature is assumed to be the true surface temperature with an added Gaussian noise of $1.5 \mathrm{~K}$. The Gaussian noise aims to simulate the accuracy of a real a priori surface temperature estimate, which can for example be obtained from AVHRR (Advanced Very High Resolution Radiometer), which together with IASI is part of the MetOp satellites payload. Here, $1.5 \mathrm{~K}$ is a very conservative assumption for tropical ocean surfaces since uncertainties in AVHRR sea surface temperature data records are typically an order of magnitude lower, e.g. estimated at $0.18 \mathrm{~K}$ in the dataset of Merchant et al. (2019).

The a priori temperature profile is assumed to be moist adiabatic up to around $100 \mathrm{hPa}$. The a priori surface temperature is used as a starting point for the moist adiabat. A moist adiabatic tropospheric temperature profile is a reasonable assumption because the temperature lapse rate is mostly set to be moist adiabatic within the tropics by deep convection and by the homogenisation of the temperature field by gravity waves due to the lack of a Coriolis force (Sobel and Bretherton, 2000). Around $100 \mathrm{hPa}$ and above, the moist adiabat is relaxed to the tropical FASCOD atmosphere with a hyperbolic tangent weight- 
ing function to represent the tropopause and the atmosphere above. The a priori $\mathrm{VMR}_{\mathrm{H}_{2} \mathrm{O}}$ profile is defined by combining a fixed relative humidity profile $(\mathrm{RH})$ and the a priori temperature profile by using the relation:

$\operatorname{VMR}_{\mathrm{H}_{2} \mathrm{O}}=\frac{\mathrm{RH}_{s}(T)}{p}$

The fixed tropical FASCOD RH profile is used and the equilibrium pressure of water vapor $e_{s}(T)$ is calculated based on the a priori temperature profile. $p$ is the atmospheric pressure in a given altitude. $e_{s}(T)$ is calculated as the equilibrium pressure over water for temperatures above the triple point and over ice for temperatures more than $23 \mathrm{~K}$ below the triple point. For intermediate temperatures the equilibrium pressure is computed as a combination of the values over water and ice according to the IFS documentation (ECMWF, 2018).

The a priori assumption about the variability of the retrieval quantities is encoded by $\mathbf{S}_{a}$, which consists of blocks for each retrieval quantity. For the surface temperature, a variance of $100 \mathrm{~K}^{2}$ is assumed. The diagonal elements of the temperature profile block of $\mathbf{S}_{a}$ (Fig. $2 \mathrm{~b}$ ) are calculated based on tropical ocean profiles from the database provided by Eresmaa and McNally (2014), which is based on the ECMWF IFS forecast model with a focus on a broad sampling of temperature profiles. The nondiagonal elements are calculated based on a correlation length that linearly increases from $2.5 \mathrm{~km}$ at the surface to $10 \mathrm{~km}$ at and above the tropopause.

For the water vapor covariances (Fig. 2c), the approach of Schneider and Hase (2011) is adapted, where the diagonal elements of the log-scale water vapor covariances are set to 1 in the troposphere and linearly reduce to 0.25 within the stratosphere. An adjustment made here is that below $2 \mathrm{~km}$, which is a crude estimate for the boundary layer height above ocean surfaces, the diagonal value linearly decreases to 0.1 at the surface. This better represents the generally fixed moisture structure near the tropical ocean surface. The nondiagonal elements are calculated based on the same correlation length approach as for the temperature covariances.

An additional constraint about the atmospheric variability is introduced by filling in values for the cross-covariances between the three retrieval quantities. The diagonals of the cross covariance blocks are calculated as the product of the diagonals of the two respective covariance blocks, multiplied with a scale factor that exponentially decreases from 1 at the surface to $1 / e$ in a given altitude. This altitude is chosen to be $100 \mathrm{~m}$ for the cross covariance between surface temperature and temperature to represent the dependence of the atmospheric temperature on the surface temperature. Between temperature and water vapor the altitude is chosen to be $1000 \mathrm{~m}$ to represent the dependence of water vapor on temperature within the boundary layer, where the water vapor content is mainly constrained by the saturation pressure, which is mainly a function of temperature. The nondiagonal elements of the cross-covariances are calculated with the same correlation length approach as for temperature and water vapor. Figure 2(a) shows the resulting cross-covariance matrix, which only has significant values within the boundary layer. 


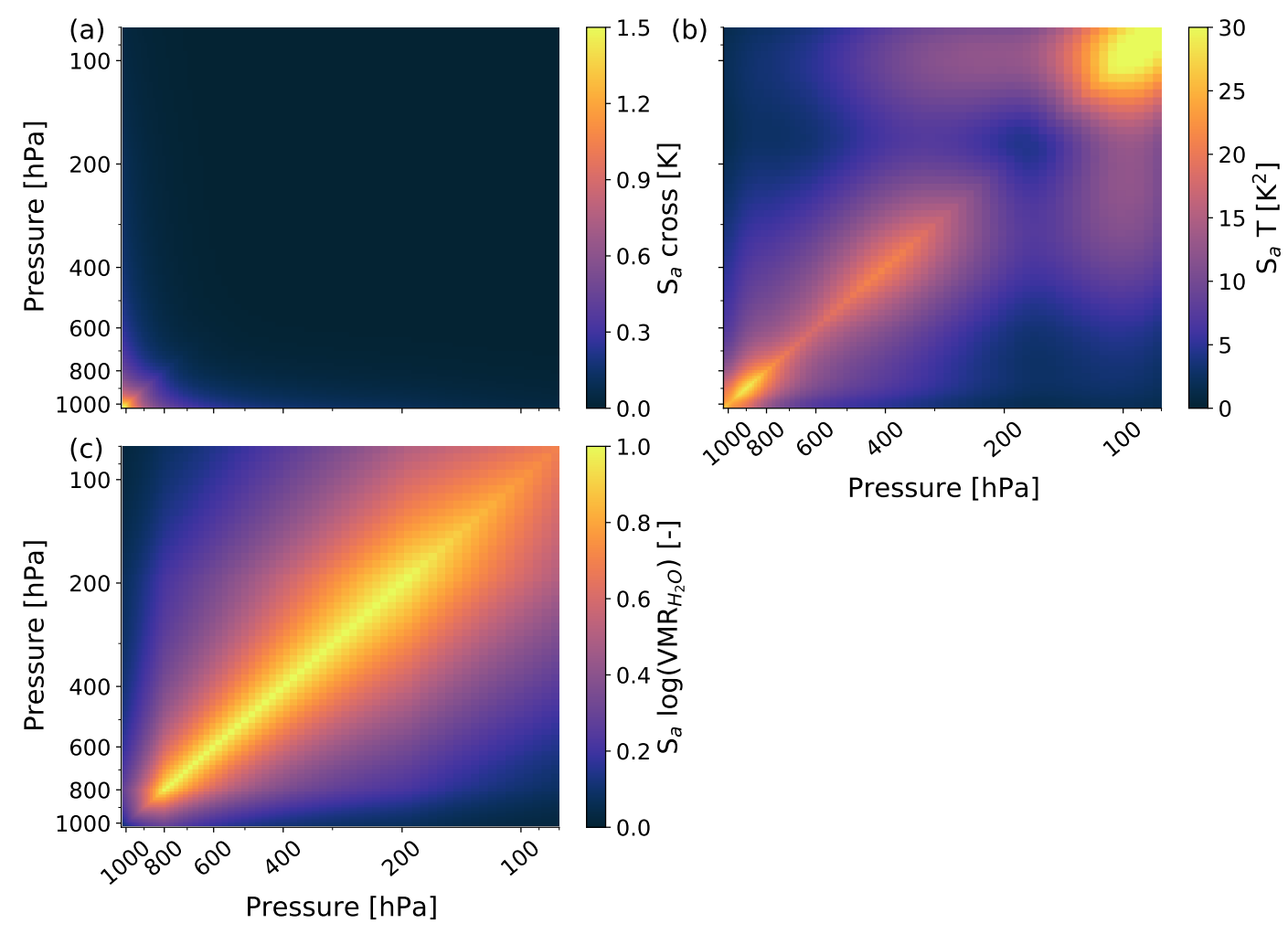

Figure 2. Covariance matrices of $\log \left(\mathrm{VMR}_{\mathrm{H}_{2} \mathrm{O}}\right)(\mathrm{c})$, temperature (b) and the cross-covariance matrix between water vapor and temperature (a) used for the retrieval in this study. Each of these matrices constitutes a block within the full covariance block matrix $\mathbf{S}_{a}$ (introduced in Eq. 3. Note that it is sufficient to show only one cross-covariance matrix block, since $\mathbf{S}_{a}$ is block symmetric.

\section{Definition and characterisation of moisture anomalies}

This section introduces a quantitative framework to identify and characterise EMLs. Besides the aim of making the term EML more graspable this way, the introduction of a number of scalar moisture anomaly characterisation metrics allows for a more targeted evaluation of retrieval results in Sect. 4 and 6.

EMLs can be described as layers of anomalously large humidity, raising the question what one implicitly assumes the

210 humidity to be increased against. Besides the actual humidity profile (be it in terms of $\mathrm{VMR}_{\mathrm{H}_{2} \mathrm{O}}$ ), one unconsciously also envisions a somewhat arbitrary climatological mean profile that smoothly decreases with height, against which the actual humidity profile partly appears anomalously moist in the presence of an EML. This idea can be used to introduce a quantitative identification and characterisation method of moisture anomalies, a subset of which are EMLs.

At the core of this moisture anomaly identification method is the definition of a reference humidity profile, against which

215 the anomalies occur. There are several ways a reference profile can be constructed and the suitability of a definition depends on the aim of the analysis. For example, a simple climatological mean profile may be a suited reference if one is interested 
in the mean anomaly (e.g. the bias) of a test dataset of humidity profiles. However, for the purpose of this study it is not of interest whether a humidity profile is generally rather moist or dry, but instead only anomalous vertical variability of humidity is of interest. This is because the vertical moisture variability is what manifests as a footprint on the heating rate profile $(Q)$ and thereby affect the vertical stability or even yield vertical motion (Albright et al., 2020).

To capture moisture anomalies closely related to the vertical moisture variability, the reference profile is constructed by least-square fitting a quadratic function to the $\log \left(\mathrm{VMR}_{\mathrm{H}_{2} \mathrm{O}}\right)$ profile of the troposphere up to $100 \mathrm{hPa}$. A quadratic function is preferable over a linear function because in many cases the $\mathrm{VMR}_{\mathrm{H}_{2} \mathrm{O}}$ profile shows large scale non-exponential variability which should not interfere with the more small-scale anomalies we want to characterise. The following function is used as the reference water vapor profile:

$\log \left(\mathrm{VMR}_{\mathrm{H}_{2} \mathrm{O}, \mathrm{ref}}\right)=a z^{2}+b z+c$

The humidity at the surface is represented by $\mathrm{VMR}_{\mathrm{H}_{2} \mathrm{O} \text {, ref }}(z=0)=\exp (c)$ and is fixed to the surface value of the actual humidity profile. The altitude $z$ is used as a height coordinate for fitting because compared to pressure it has the benefit that $z=0$ at the surface. The coefficients $a$ and $b$ are determined by least-square fitting to the logarithm of the humidity profile between the surface and $100 \mathrm{hPa}$ because the assumed relation becomes less valid closer to the tropopause. After calculating the reference profile, moisture anomalies can be identified and characterised.

To visualise the moisture anomaly identification and characterisation procedure, Fig. 3(a) depicts an exemplary case with a strong EML peaking at around $650 \mathrm{hPa}$. The atmospheric test case used here is constructed to be similar to the EML case investigated by Stevens et al. (2017). The testcase is constructed based on the tropical FASCOD atmosphere (Anderson et al., 1986). The VMR of water vapor and the temperature profiles of the tropical FASCOD atmosphere are modified to represent the typical structures associated with an EML scenario. The EML associated structures include a distinct moisture inversion (increase of $\mathrm{VMR}_{\mathrm{H}_{2} \mathrm{O}}$ with height) with maximum humidity at around $650 \mathrm{hPa}$ and a temperature inversion at the EML top. Another temperature inversion is located at the distinct drop of moisture at around $900 \mathrm{hPa}$, similar to the case investigated by Stevens et al. (2017).

Figure 3(b) shows the close relation between the vertical humidity structure and the net heating rate $Q$ (longwave + shortwave), which is calculated with the radiative transfer model RRTMG (Rapid Radiative Transfer Model for GCMs, Mlawer et al., 1997) through its implementation in the radiative convective equilibrium model konrad (Kluft and Dacie, 2020). $Q$ is calculated for all conducted retrievals throughout this study to assess whether the vertical humidity structure is captured in a way in which also $Q$ is represented well.

The blue and orange shading associated with moist and dry anomalies depicted in Fig. 3 visualises that by definition layers of positive and negative moisture anomalies alternate in the vertical. Each such layer can be viewed as a moisture anomaly object, which we characterise by means of the scalar metrics introduced in table 1 . These metrics include the vertical bounds of the moisture anomaly in terms of altitude $\left(z_{\text {bot }}\right.$ and $\left.z_{\text {top }}\right)$, the difference of which denotes the anomaly's thickness $\left(\Delta z_{\text {anom }}\right)$. The anomaly height $\left(z_{\mathrm{anom}}\right)$ is defined as the mean over the anomaly's height interval, weighted by the anomalous humidity within 
https://doi.org/10.5194/amt-2021-48

$\begin{array}{r}\text { Atmospheric } \\ \text { Measurement } \\ \text { Techniques } \\ \hline \text { Discussions }\end{array}$

(c) Author(s) 2021. CC BY 4.0 License.

(c) (1)

250 the altitude bounds. Finally, the anomaly strength $\left(s_{\text {anom }}\right)$ is defined as the mean anomalous $\mathrm{VMR}_{\mathrm{H}_{2} \mathrm{O}}$ within the anomaly's vertical bounds. We only consider positive (moist) anomalies that are fully captured in the pressure range between 100 and $900 \mathrm{hPa}$, e.g. the positive anomalies at the very top and bottom of Fig. 3 are neglected (grey shading) to avoid tropopause and boundary layer related anomalies.
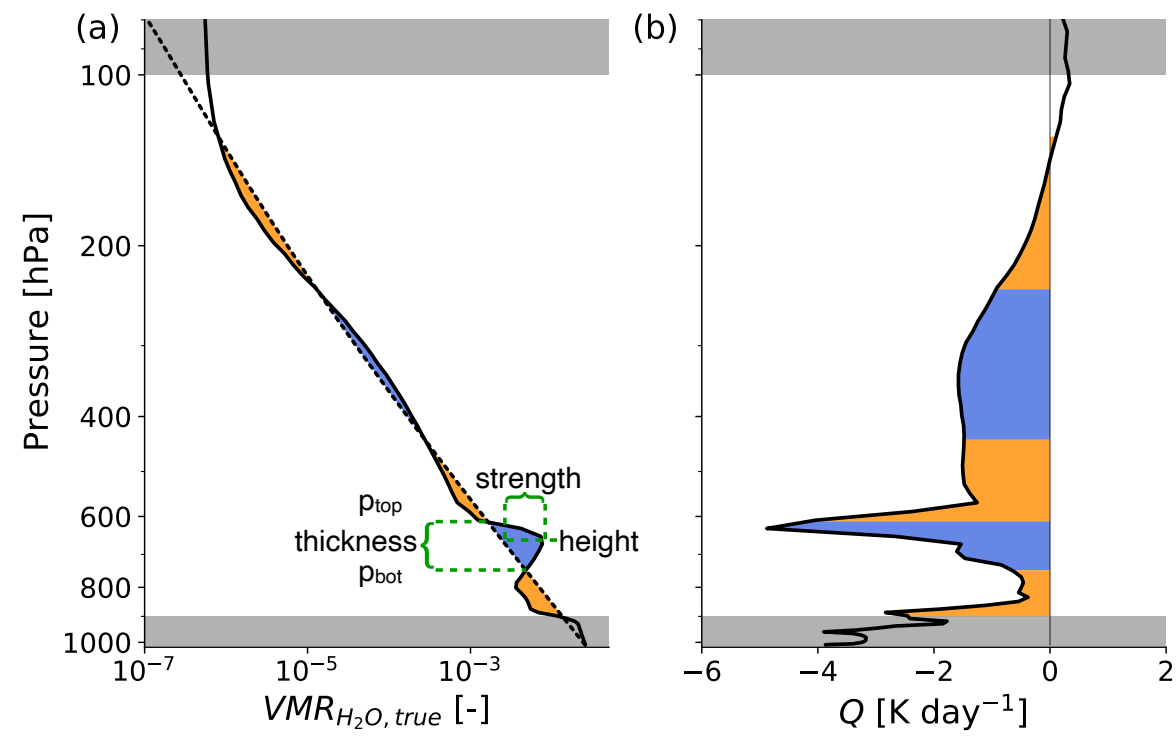

Figure 3. Exemplary humidity profile (a) of an atmospheric case with a strong EML and the associated net heating rate (longwave + shortwave) profile (b). The reference humidity profile used to identify humidity anomalies is depicted as the dashed line in (a). Layers of moist anomalies are highlighted by blue shading, dry anomalies by orange shading. Anomalies that intersect with the grey shaded regions are excluded to restrict anomalies to the free troposphere. The green lines and brackets conceptually display the definition of moisture anomaly characteristics from table 1 for the strong positive anomaly at around $650 \mathrm{hPa}$. 
Table 1. Characterisation metrics of moisture anomalies, their definitions and short descriptions. VMR $\mathrm{H}_{2} \mathrm{O}$, ref denotes the reference water vapor profile.

\begin{tabular}{|c|c|c|}
\hline Metric & Definition & Description \\
\hline Bounds & $z_{\mathrm{bot}}, z_{\mathrm{top}}$ & Lower and upper altitude bounds of the anomaly. \\
\hline Thickness & $\Delta z_{\mathrm{anom}}=z_{\mathrm{top}}-z_{\mathrm{bot}}$ & Altitude interval of the anomaly. \\
\hline Strength & $s_{\text {anom }}=\frac{1}{z_{\text {top }}-z_{\text {bot }}} \int_{z_{\text {bot }}}^{z_{\text {top }}}\left(\mathrm{VMR}_{\mathrm{H}_{2} \mathrm{O}}-\mathrm{VMR}_{\mathrm{H}_{2} \mathrm{O}, \text { ref }}\right) d z$ & Mean anomalous $\mathrm{VMR}_{\mathrm{H}_{2} \mathrm{O}}$ within the anomaly. \\
\hline Height & $z_{\text {anom }}=\frac{\int_{z_{\text {bot }}}^{z_{\text {top }}} z\left(\mathrm{VMR}_{\mathrm{H}_{2} \mathrm{O}}-\mathrm{VMR}_{\mathrm{H}_{2} \mathrm{O}, \mathrm{ref}}\right) d z}{\int_{z_{\mathrm{bot}}}^{z \text { top }}\left(\mathrm{VMR}_{\mathrm{H}_{2} \mathrm{O}}-\mathrm{VMR}_{\mathrm{H}_{2} \mathrm{O}, \mathrm{ref}}\right) d z}$ & Weighted mean altitude of the anomaly. \\
\hline
\end{tabular}

\section{Case study of a moist layer retrieval}

In this section the retrieval is applied to the atmospheric scenario introduced in Sect. 3, which includes an EML. This allows for a first assessment of whether or not the retrieval appears capable of resolving the EML. The importance of temperature information for the retrieval to resolve the moisture structure is assessed by also applying an altered retrieval setup that does not retrieve the temperature profile. In a next step, the averaging kernels for the EML scenario and a mean tropical ocean atmosphere are compared to estimate the retrieval's vertical resolution and its dependence on the atmospheric state.

\subsection{Importance of temperature information for the humidity retrieval}

To get an exemplary impression of the retrieval's ability to resolve an EML, we apply it to the atmospheric test case introduced in Sect. 3, which is similar to the case that originally showed the satellite blind spot in Stevens et al. (2017). By adjusting the retrieval's spectral setup to the setup of Stevens et al. (2017) and omitting the temperature profile retrieval, the possibility of an EML blind spot associated with these retrieval setup properties is investigated. The test case profiles to be retrieved are displayed in Fig. 4 as the profiles denoted as "true". Based on these profiles, forward simulated synthetic IASI observations are calculated, synthetic Gaussian noise is added (see Sect. 2.5) and the retrieval is performed.

The upper row panels in Fig. 4 show the retrieval results based on the setup introduced in Sect. 2, from now on referred to as retrieval setup 1. The lower row of panels in Fig. 4 shows results based on an altered retrieval setup that deviates from setup 1 by not explicitly retrieving the temperature profile and using a more narrow spectral region, which is limited to 1193 to 1223 and 1251 to $1253 \mathrm{~cm}^{-1}$. This setup is from now on referred to as retrieval setup 2. Retrieval setup 2 is based on some of the retrieval setup properties used by Lacour et al. (2012) and later also by Stevens et al. (2017). Note that the retrieval results of Stevens et al. (2017) are not aimed to be comprehensively reproduced in this study, but rather used as a starting point to 
assess the possibility of an EML blind spot for IASI. The major difference between retrieval setups 1 and 2 is the way in which the vertical temperature structure is represented in the retrieval, which we hypothesise to be the key for resolving moisture structures such as EMLs. While retrieval setup 2 is based solely on a priori temperature information, retrieval setup 1 is aimed at optimally resolving the temperature structure by making use of the independent temperature information available from the $\mathrm{CO}_{2}$ band between 2150 to $2400 \mathrm{~cm}^{-1}$.
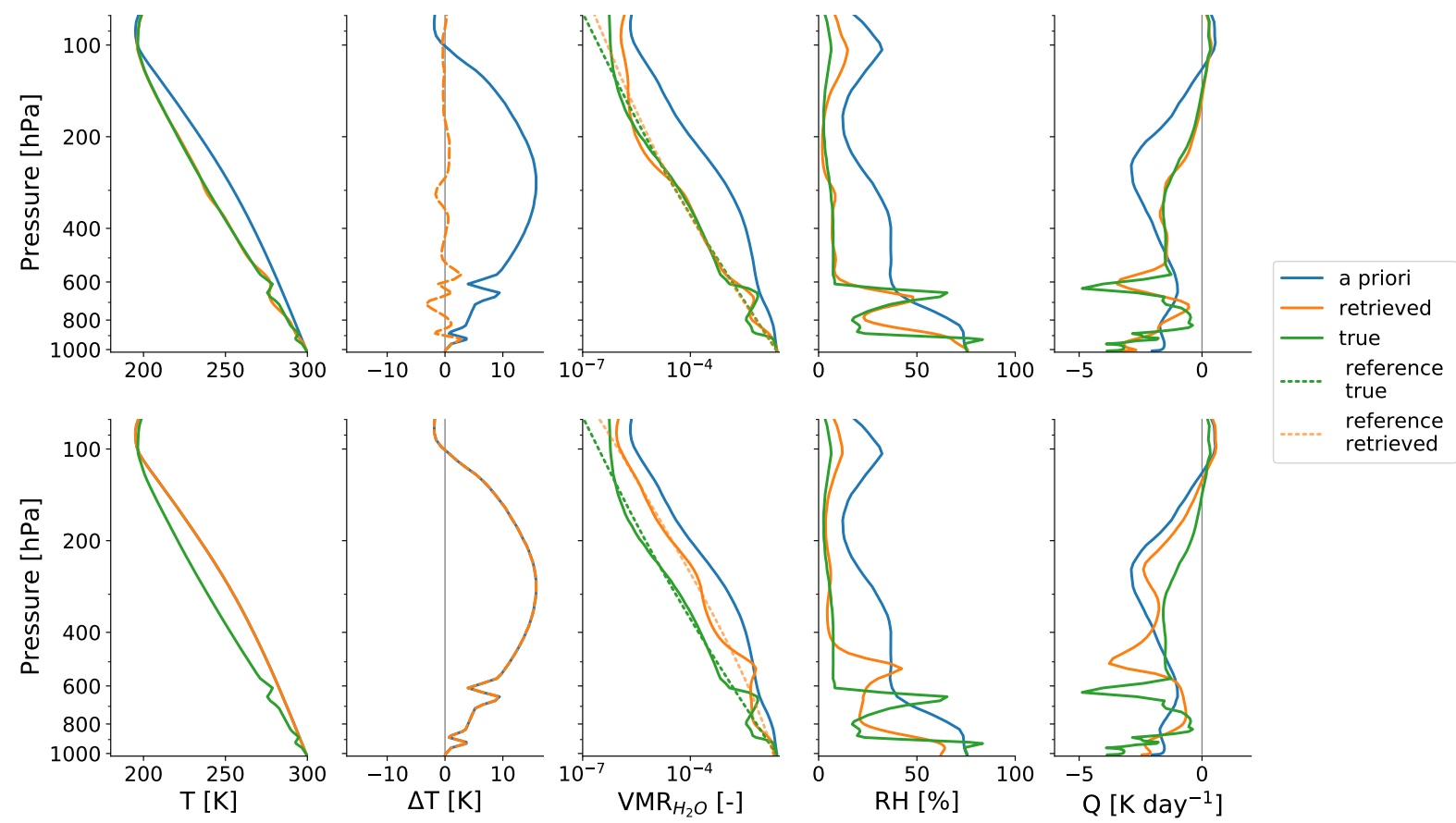

reference retrieved

Figure 4. Profiles of temperature $(T)$, temperature differences of a priori and retrieved states to the true state $(\Delta T), \mathrm{VMR}_{\mathrm{H}_{2} \mathrm{O}}$, relative humidity $(\mathrm{RH})$ and net heating rates $(Q)$ for the EML test case introduced in Sect. 3 (labeled as "true" here). Displayed are the true states that are used as a basis for the forward modelled synthetic IASI observation, the a priori states of the retrieval and the retrieved states. Upper panels show results of retrieval setup 1 and lower panels of retrieval setup 2, which are introduced in the text.

Looking at the retrieval results in the upper row of Fig. 4, the EML structure is generally found to be resolved well with retrieval setup 1, albeit that the retrieved EML is less pronounced. This is reflected in table 2, which shows the calculated moisture anomaly characteristics introduced in Sect. 3 for the EML in around $650 \mathrm{hPa}$ in the true state and for the according anomaly found by the two different retrieval setups. It shows that the EML strength $s_{\text {anom }}$ of retrieval setup 1 is about half the value of the true state. While the EML height $\left(z_{\text {anom }}\right)$ is captured well, the EML is found to be about $600 \mathrm{~m}$ thicker with retrieval setup 1 . The temperature profile in the upper and mid troposphere is resolved well with retrieval setup 1, even capturing the general structure of the temperature inversion at the EML top. The second temperature inversion above the boundary layer is not resolved, which we mainly attribute to the fact that no independent temperature information is available for these altitudes, e.g. there are no sufficiently weak $\mathrm{CO}_{2}$ lines. However, for the retrieval of the EML, these altitudes are not important. 
Table 2. Moisture anomaly characteristics of the EML in the exemplary testcase shown in Fig. 4. The EML characteristics are calculated for the true state and the retrieval results of the two retrieval setups, corresponding to upper and lower rows of Fig. 4, respectively.

\begin{tabular}{ccccc}
\hline Metric & Variable name & True state & Retrieval setup 1 & Retrieval setup 2 \\
\hline Bounds & $z_{\text {bot }}, z_{\text {top }}$ & $2.8 \mathrm{~km}, 4.3 \mathrm{~km}$ & $2.8 \mathrm{~km}, 4.8 \mathrm{~km}$ & $4.3 \mathrm{~km}, 6.7 \mathrm{~km}$ \\
Thickness & $\Delta z_{\text {anom }}$ & $1.5 \mathrm{~km}$ & $2.1 \mathrm{~km}$ & $2.4 \mathrm{~km}$ \\
Strength & $s_{\text {anom }}$ & $2.9610^{-3}$ & $1.5910^{-3}$ & $2.0610^{-3}$ \\
Height & $z_{\text {anom }}$ & $3.4 \mathrm{~km}$ & & $3.5 \mathrm{~km}$ \\
\hline
\end{tabular}

For retrieval setup 2, table 2 shows that the retrieved EML is a bit stronger than with retrieval setup 1, but is located about $2 \mathrm{~km}$ above the true state EML. This is where the importance of a proper representation of the temperature profile for the humidity retrieval becomes apparent. The erroneous placement of the EML with retrieval setup 2 is the most apparent manifestation of a temperature induced error in the retrieved water vapor profile. Since the temperature profile is not retrieved with setup 2, signals associated with the temperature profile are erroneously interpreted as signals of water vapor. This effect is very apparent given the strong temperature bias of the a priori. This is avoided with retrieval setup 1 where the temperature profile is explicitly retrieved. It is plausible that unresolved smaller scale temperature structures also interfere with the retrieval's ability to capture humidity structures of similar scale. However, based on the retrieval results of setup 2, a lack of temperature information alone does explain the absence of the EML in the retrieval, as found by Stevens et al. (2017).

The main conclusion of this experiment is that EMLs generally do not appear to be a blind spot for passive hyperspectral infrared satellite observations. Setup 1 appears significantly more suited for capturing EMLs reliably than setup 2 due to its better representation of the vertical temperature structure. We argue that the apparent EML blind spot found by Stevens et al. (2017) is not a fundamental limitation, but rather a result of the particular retrieval setup that was applied.

\subsection{Retrieval resolution}

Besides the more phenomenological assessment of the retrieval's ability to resolve an EML in the previous section, OEM (Sect. 2.3) allows for a more quantitative estimation of the vertical scales the retrieval is able to resolve. The averaging kernel matrix A is a commonly used quantity to estimate the vertical resolution of an observing system and can be calculated as (Rodgers, 2000): 
The rows of $\mathbf{A}$ describe the response of the retrieved state to a perturbation in the true state, taking into account the specifications of the observing system represented by $\mathbf{K}$ and $\mathbf{S}_{y}$ and the a priori assumption on the retrieval quantities variability represented by $\mathbf{S}_{a}$.

Figures 5(a) and (c) depict the rows of the $\mathrm{H}_{2} \mathrm{O}$ averaging kernel matrix as colored lines for two different atmospheric setups. The more blue lines correspond to kernels closer to the surface, while the more yellow lines correspond to kernels higher up in the atmosphere. Figures 5(a) and (b) are based on an average tropical ocean atmosphere, namely the tropical FASCOD atmosphere introduced in Sect. 2.5. Figures 5(c) and (d) only differ in their base atmospheric state by the introduced EML, as described in Sect. 3. The vertical width of a kernel is a measure of retrieval's vertical resolution in a specific height, which is shown in terms of Full Width at Half Maximum (FWHM) of the respective kernels in Fig. 5(b) and (d). A measure for the retrieval's ability to capture a water vapor signal in a given height is the measurement response, which is defined as the sum over all kernel rows and depicted as the black line in Fig. 5(a) and (c). Values close to 1 indicate a good sensitivity of the retrieval.

The averaging kernels of the mean tropical ocean atmosphere in Fig. 5(a) expectably show a very smooth behaviour with height. Their FWHM values is on the order of $1.5 \mathrm{~km}$ throughout most of the free troposphere, e.g. from around 200 to $800 \mathrm{hPa}$. In the upper troposphere $(p \lesssim 200 \mathrm{hPa})$ a significant decrease in vertical resolution is found. In the boundary layer, the vertical resolution does not appear to diminish, which is misleading because the shape of the averaging kernels associated with these altitudes is distorted due to the strong signal of the surface. Rather than the FWHM, the measurement response is a more informative measure of retrievals sensitivity to disturbances in the boundary layer. For the tropical ocean atmosphere, the measurement response is close to one throughout most of the free troposphere and shows a sharp decrease within the boundary layer, indicating limited sensitivity to water vapor anomalies only in the boundary layer.

The EML has a significant impact on all averaging kernels in the lower and mid troposphere as shown in Fig. 5(c) and (d). Around the humidity maximum at the EML top it is clear that the averaging kernels show distinct peaks, which are caused by the strong radiative signal associated with the EML. The EML signal is so strong that it also affects the more sensitive channels that usually sample higher altitudes and therefore decreases the vertical resolution from about $1.5 \mathrm{~km}$ to $2 \mathrm{~km}$ between the EML top and about $200 \mathrm{hPa}$ compared to the tropical mean atmosphere. Below the EML top the decrease in moisture towards lower altitudes goes along with a clear reduction in vertical resolution to about $2.4 \mathrm{~km}$ at around $800 \mathrm{hPa}$, indicating a more limited ability to resolve additional moisture features below the EML. This state dependence of the averaging kernel reflects the nonlinear nature of the retrieval problem and the limited expressiveness of the vertical resolution deduced with this method. However, the presented numbers on vertical resolution suffice as a first quantitative estimate on what scales the retrieval is able to resolve. As a next step, a statistical evaluation of the retrieval's performance is conducted to assess the limitations in vertical resolution and specifically to retrieve moisture anomalies. 

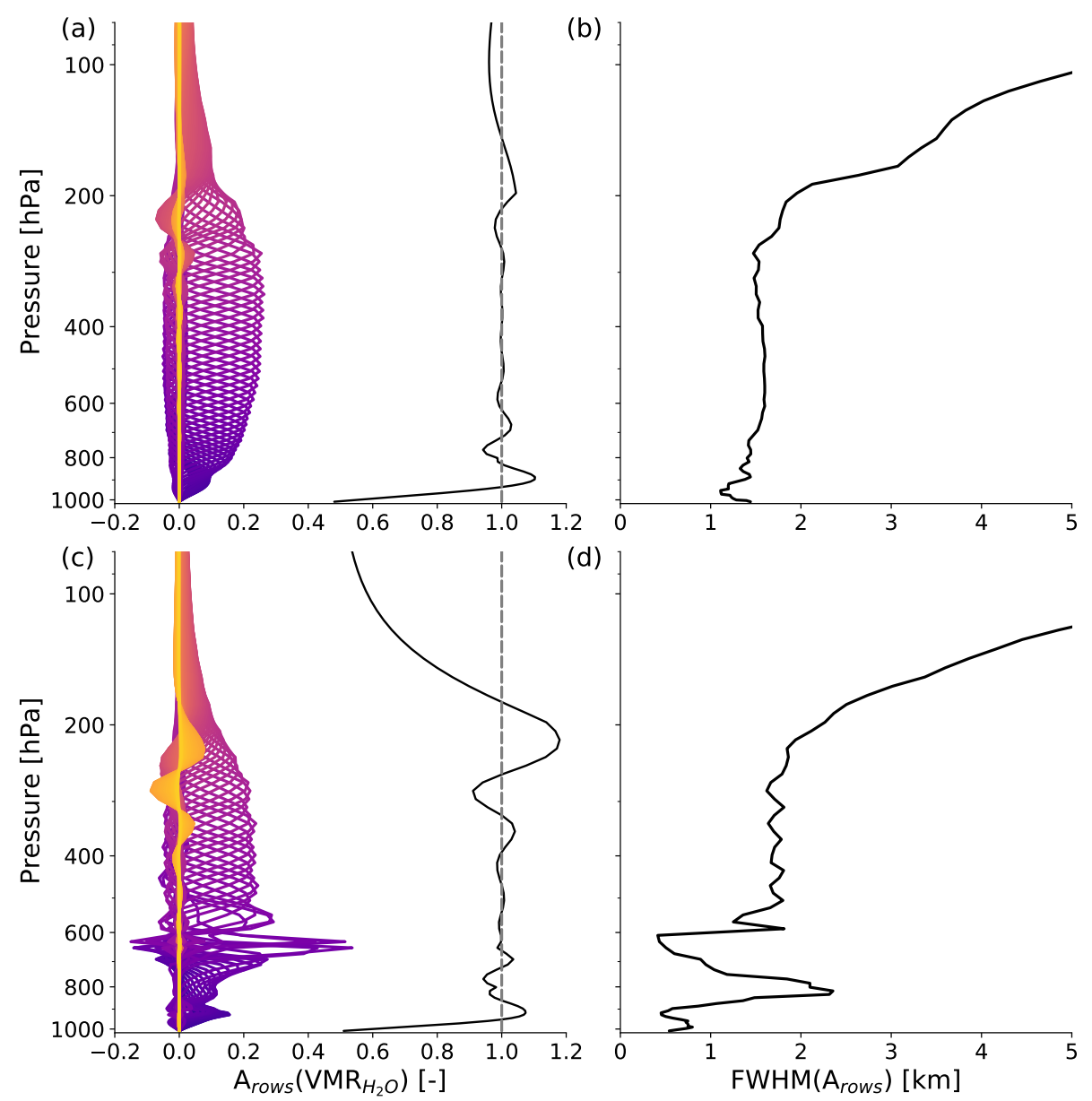

Figure 5. (a) and (c) show rows of the water vapor averaging kernel matrix $\left(A_{\text {rows }}\left(\mathrm{VMR}_{\mathrm{H}_{2} \mathrm{O}}\right)\right)$ as colored lines and their sum as a black line, which denotes the measurement response. The rather blue lines correspond to kernels closer to the surface, the more yellow lines correspond to kernels in higher altitudes. (b) and (d) show the FWHM of the averaging kernel rows, which is a measure for the vertical resolution of the observing system. (a) and (b) are based on a mean tropical ocean atmosphere, specifically the tropical FASCOD atmosphere. The atmospheric setup used for (c) and (d) differs only by the introduction of EML features, as described in Sect. 3.

\section{Retrieval performance}

After the investigation of an exemplary EML testcase in the previous section, the retrieval performance is now assessed based on a larger test dataset. First, the test dataset is introduced and the vertical distribution of the retrieval error in temperature and water vapor is investigated. Afterwards, the smoothing error (SE), which is an intrinsic source of error for a given observing system and a set of a priori assumptions, is calculated and discussed in the context of the overall retrieval error. 


\subsection{Reference dataset and retrieval error}

The retrieval is applied to tropical ocean atmospheres (between $30^{\circ} \mathrm{S}$ to $30^{\circ} \mathrm{N}$ ) that are part of the ECMWF IFS diverse profile database made available by Eresmaa and McNally (2014). The database consists of 25,000 short-range forecasts, which are divided into five even subsets that focus on representing diversity in a particular atmospheric quantity, such as temperature, specific humidity or precipitation. For the purpose of this work, only the tropical ocean atmospheres of the subset that focuses on a diverse sampling of specific humidity is considered. This yields a total number of 1599 atmospheric setups, for 1438 of which the retrieval converges to a solution. The following analysis is based on these converged cases.

A statistical overview of the variety of temperature and humidity profiles covered by the tropical ocean dataset is provided in Fig. 6(a), (b) and (c). The temperature profiles show very limited variability, as is typical for tropical ocean regions. However, despite this very smooth appearance of the vertical temperature structure, the individual profiles do include significant temperature inversions, for example the very prominent inversion in about $2 \mathrm{~km}$ height in the trade wind region (not shown). The humidity profiles show weak variability within the boundary layer, where the ocean acts as a humidity source and humidity is mostly set by the saturation vapor pressure controlled by temperature. The median $\mathrm{RH}$ is about $82 \%$ at the surface and reaches its maximum in about $500 \mathrm{~m}$ height in the transition to the shallow cloud layer. In the free troposphere, the typical " $\mathrm{C}$ " shape structure of the RH profile is followed (Romps, 2014). An interesting feature in the 75th and 90th percentiles of the RH profiles is the presence of positive $\mathrm{RH}$ anomalies in the layer between around 500 and $700 \mathrm{hPa}$, indicating moisture anomalies that may be tied to the freezing level.

Figures 6(d), (e) and (f) show an overview of the retrieval's deviations from the reference dataset, from now on referred to as the retrieval error. In the context of these figures, the term bias refers to a difference of the median values of the retrieved and the true datasets. The temperature profile shows a positive bias close to the surface, which we attribute to the limited signal from these heights in the satellite observation. The negative bias near the surface in $\mathrm{RH}$ is on the one hand associated with this positive temperature bias and on the other hand with the slightly negative $\mathrm{VMR}_{\mathrm{H}_{2} \mathrm{O}}$ bias near the surface. Between around 900 to $700 \mathrm{hPa}$ the $\mathrm{VMR}_{\mathrm{H}_{2} \mathrm{O}}$ and $\mathrm{RH}$ biases are positive, while the temperature bias is slightly negative. This positive moisture bias in the lower troposphere is associated with an increased variability of the error, particularly towards strong positive errors that indicate an overestimation of moisture in the lower troposphere by the retrieval. This may be caused by the typical hydrolapse that is coupled to the trade inversion in the trade wind regions, which can in its sharpness not be captured by the retrieval.

In the mid troposphere between about 700 to $300 \mathrm{hPa}$, which is where typical EMLs are expected, no significant temperature or humidity biases are found. A positive skewness in the $\mathrm{VMR}_{\mathrm{H}_{2} \mathrm{O}}$ error distribution towards strong positive errors is found, indicating that positive errors in retrieved $\mathrm{VMR}_{\mathrm{H}_{2} \mathrm{O}}$ are rare, but large compared to the negative errors that occur. As an explanation for this error pattern, we propose the idea that positive (moist) moisture anomalies tend to be captured with a slight underestimation in their strength, while occasionally strong negative (dry) moisture anomalies beneath are associated with a strong overestimation of moisture by the retrieval due to a lack of signal beneath a positive moisture anomaly (as shown in Fig. 5). This could explain less frequent but strong positive retrieval errors and more frequent, but relatively weak negative errors that have a net bias close to zero. 
In the upper troposphere errors in temperature and humidity are generally larger. We believe that this has two causes. On the one hand the a priori moist adiabatic temperature assumption becomes worse closer to the tropopause. On the other hand, Fig. 5 shows that there is only a weak radiative signal from the upper troposhere as indicated by strongly smoothed averaging kernels and a decreased vertical resolution. While this may be improved by adjusting the a priori assumptions for the upper troposphere and including even stronger absorption features of water vapor, the upper troposphere is no major concern of this study.
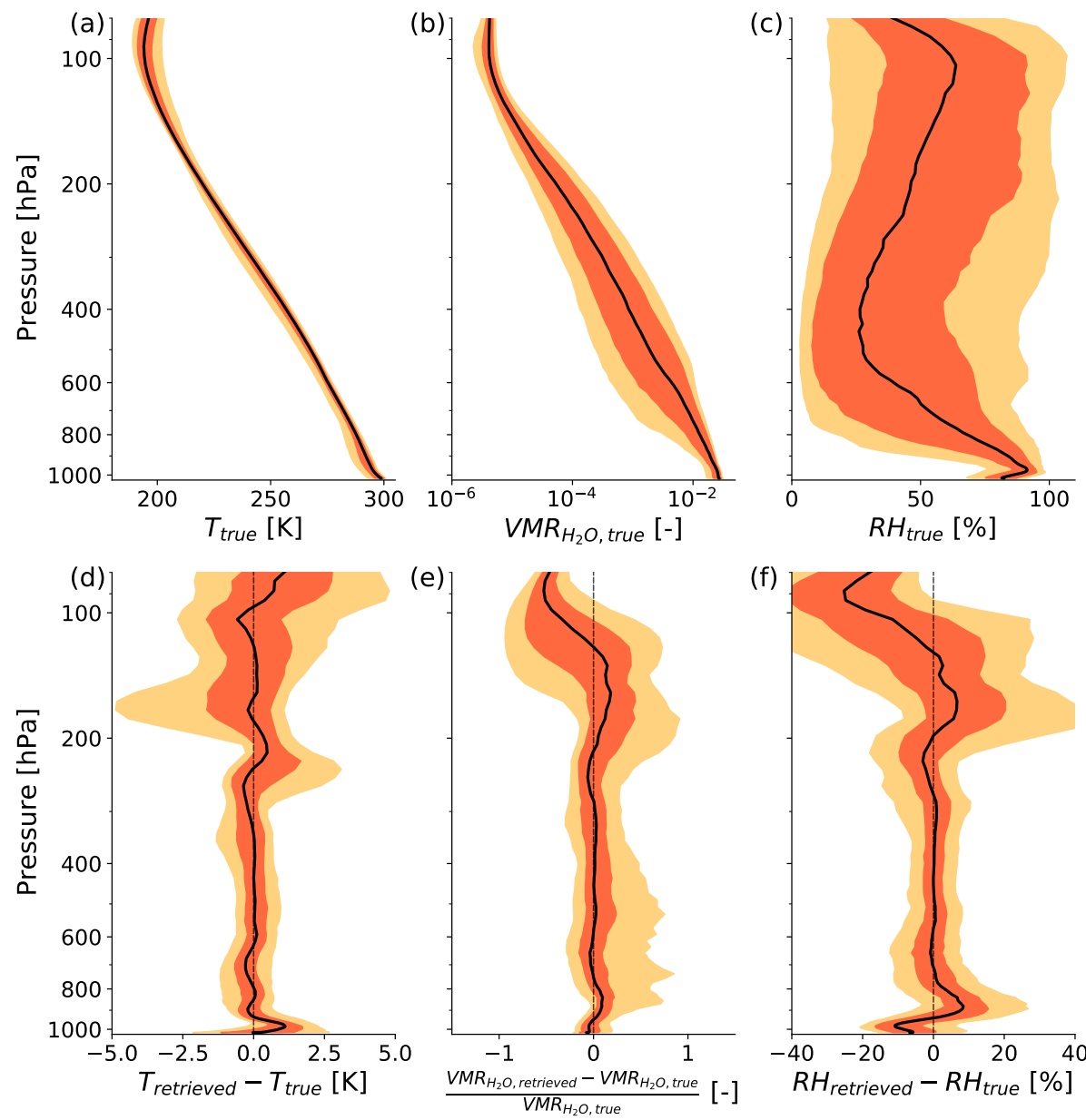

Figure 6. (a), (b) and (c) give a statistical overview of temperature, $\mathrm{VMR}_{\mathrm{H}_{2} \mathrm{O}}$ and $\mathrm{RH}$ over 1438 tropical ocean model atmospheres from the dataset of Eresmaa and McNally (2014), upon which the retrieval is performed. Color scheming is based on Figure 5 of Eresmaa and McNally (2014), where bright orange indicates 10th and 90th percentiles, dark orange indicates 25 th and 75 th percentiles. The median is depicted by a solid black line. (e), (f), (g) show the respective statistics on the deviations of the retrieved to the true profiles. Note the exception of relative differences for $\mathrm{VMR}_{\mathrm{H}_{2} \mathrm{O}}$, which is more suited for the dynamical range of this quantity. 


\subsection{Smoothing error}

Part of the retrieval error shown in Fig. 6 can be attributed to the so called Smoothing Error (SE, Rodgers 2000). Given a specific observing system and a priori assumptions about the quantity to be observed, the SE is a source of error that can not be avoided without changing the observing system or a priori assumptions themselves. In the frame of the averaging kernel matrix, the SE expresses the error in the retrieval that is associated with the non-delta-function shape of the averaging kernel rows (see Fig. 5) and the thereby limited ability to resolve vertical features. Here, it is calculated as

$\boldsymbol{S E}=\left(\boldsymbol{x}-\boldsymbol{x}_{a}\right)\left(\mathbf{A}-\mathbf{I}_{n}\right)$

where $\mathbf{I}_{n}$ denotes the identity matrix of order $n$ and $n$ is the number of vertical levels of the profile retrieval.

Figure 7 shows the SE statistics associated with the retrieved temperature and humidity profiles of the tropical ocean dataset. The median of the $\mathrm{SE}$ with respect to the temperature profile $(\mathrm{SE}(T))$ is close to zero throughout most of the free troposphere, similar to the retrieval bias shown in Fig. 6(d). The positive retrieval bias in temperature found near the surface is with smaller magnitude also found in $\mathrm{SE}(T)$, indicating that this pattern is caused by a systematically unresolved vertical feature. The variability of the temperature retrieval error found in Fig. 6(d) in the lower and mid troposphere cannot be attributed to smoothing, since the variability in $\mathrm{SE}(T)$ is very small. In conclusion, this indicates that temperature error sources are unlikely to be caused by uncaptured vertical temperature variability, but rather vertically constant biases, which do not show up in $\operatorname{SE}(T)$. In the upper troposphere, $\mathrm{SE}(T)$ increases towards the tropopause where smoothing becomes the major contribution to the retrieval temperature error.

For the water vapor profile in the lower and mid troposphere, smoothing is a greater source of error than for the temperature profile (Fig. 7b). While the median of the water vapor smoothing error $\left(\mathrm{SE}\left(\log \left(\mathrm{VMR}_{\mathrm{H}_{2} \mathrm{O}}\right)\right)\right)$ is low throughout the lower and mid troposphere, its variability (e.g. the shown percentile ranges) is on similar scale as the variability of the retrieval error shown in Fig. 6(e). This indicates that a major contribution of error in the water vapor retrieval is to capture vertical variability. The distribution of $\mathrm{SE}\left(\log \left(\mathrm{VMR}_{\mathrm{H}_{2} \mathrm{O}}\right)\right)$ in the mid troposphere also reflects the positive skewness that was found in the overall error in Fig. 6(e). This is consistent with the previously described idea that this skewness is linked to the retrieval's ability of capturing vertical moisture anomalies. In the upper troposphere, the median $\mathrm{SE}\left(\log \left(\mathrm{VMR}_{\mathrm{H}_{2} \mathrm{O}}\right)\right)$ increases to a similar magnitude as the retrieval error, while its variability even exceeds that of the retrieval error, indicating that other sources of error are compensating.

The SE $(\mathrm{RH})$ statistics show the combined effect of the smoothing errors in temperature and humidity (Fig. 7c). It is apparent that also in terms of RH the smoothing error has a strong contribution to the retrieval error in the lower and mid troposphere, similar to the $\mathrm{VMR}_{\mathrm{H}_{2} \mathrm{O}}$ error. In the upper troposphere the median $\mathrm{SE}(\mathrm{RH})$ is on the same order as the retrieval error, while its variability appears to be even stronger, following the behaviour found for $\mathrm{SE}\left(\log \left(\mathrm{VMR}_{\mathrm{H}_{2} \mathrm{O}}\right)\right)$. 

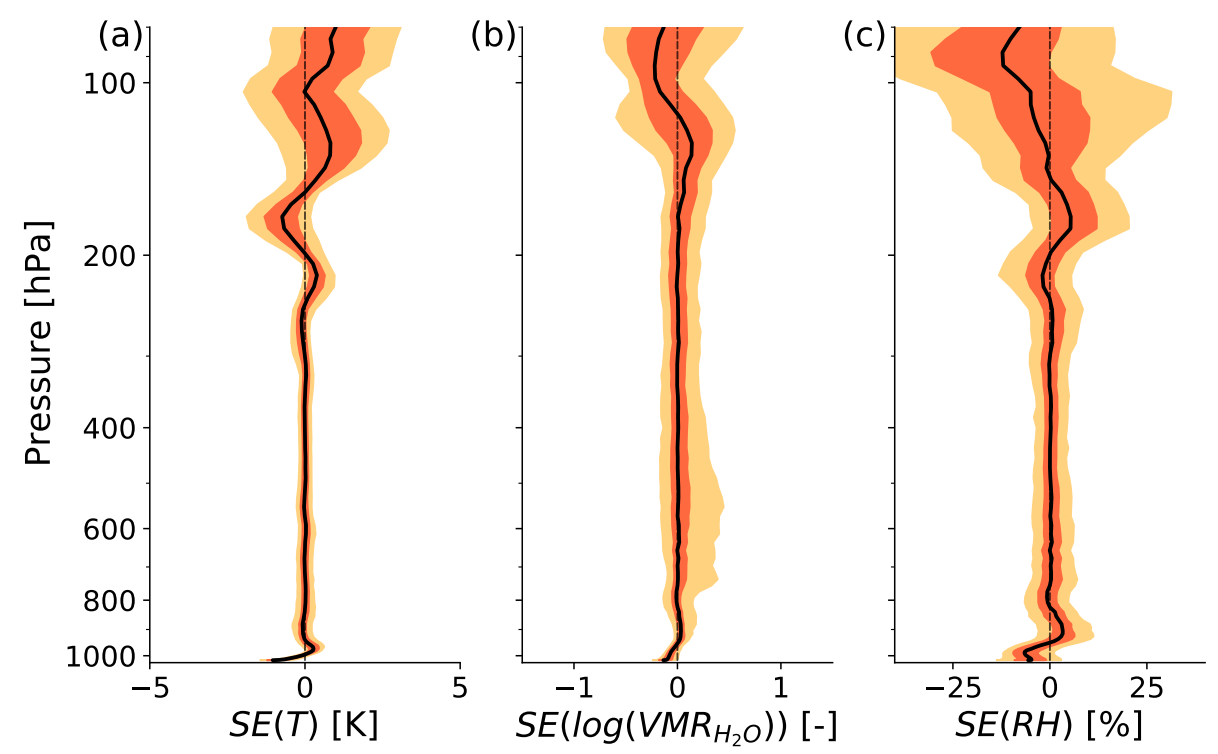

Figure 7. Smoothing Error (SE) as calculated by Eq. 7 of retrieved temperature (a), logarithmic $\mathrm{VMR}_{\mathrm{H}_{2} \mathrm{O}}$ (b) and RH (c) profiles over 1438 tropical ocean atmospheres. Lines and shadings are defined as in Figure 6.

\section{Retrieval of moisture anomalies}

In this section the retrieval results for the previously introduced tropical ocean test dataset (Sect. 5) are assessed with specific focus on the characteristics of moisture anomalies as introduced in Sect. 3. First, the moisture anomaly characteristics of the tropical ocean dataset and of the retrieved dataset are compared to look for systematic limitations of the retrieval to resolve specific kinds of moisture anomalies. Then, the impact of moisture anomalies on the heating rate profile is assessed and the retrieval's ability to capture this impact is investigated.

\subsection{Moisture anomaly characteristics}

Figure 8 shows probability density distributions of the moisture anomaly characteristics (defined in Sect. 3) for the tropical ocean dataset (green) and the associated retrieved dataset (orange). The dashed lines indicate the mean values of the respective distributions. The distributions of moisture anomaly height $\left(z_{\text {anom }}\right)$ displayed in Fig. 8(a) show that most moisture anomalies occur in the mid to upper troposphere, which is somewhat surprising since EMLs are typically thought to be coupled to the freezing level in around $5 \mathrm{~km}$ height (Johnson et al., 1996; Stevens et al., 2017). However, note on the one hand that strong EMLs and very slight moisture anomalies are treated evenly here. On the other hand the distributions reflect the statistics of the underlying dataset, which is based on the ECMWF IFS atmospheric model. This dataset appears as a suitable starting point to assess the retrieval's ability to capture moisture anomalies, however, the analysis of the dataset's moisture anomaly statistics themselves are not the focus of this study. 
Figure 8(a) shows a bias between true and retrieved $z_{\text {anom }}$ of about $1.1 \mathrm{~km}$, which is caused by an underestimation of the fraction of moisture anomalies below $5 \mathrm{~km}$ altitude by the retrieval, while the fraction of anomalies above $10 \mathrm{~km}$ is overestimated. Only about $30 \%$ of the total amount of moisture anomalies below $5 \mathrm{~km}$ in the reference dataset are captured by the retrieval. We attribute this deficiency to the fact that moisture anomalies are typically more narrow in the lower to mid troposphere than further aloft, as shown in Fig. 9.

Figure 9(a), (b) and (c) show the number of moisture anomalies of the reference dataset in the lower, mid and upper troposphere, respectively, as a function of anomaly width $\left(\Delta z_{\text {anom }}\right)$ and separated into subsets of anomalies that either could or could not be retrieved. An anomaly of the reference dataset is considered not retrieved, if there is no retrieved positive moisture anomaly with an anomaly height within the vertical bounds of the anomaly of the reference dataset. While it is apparent that the more narrow moisture anomalies are most frequently missed in all three altitude regions, this means a particular shortcoming for the retrieval between 0 to $5 \mathrm{~km}$ because cases with $\Delta z_{\text {anom }} \gtrsim 2 \mathrm{~km}$ are especially rare. This finding is coherent with the idea that the lower to mid troposphere is subject of more small-scale variability due to its link to the boundary layer and low level convection. Additionally, the adiabatic expansion of an anomalously moist air parcel as it moves up the troposphere will increase the anomaly's thickness higher up in the troposphere.

The distribution of the moisture anomaly strength $\left(s_{\text {anom }}\right)$ depicted in Fig. $8\left(\right.$ b) has a similar dynamical range as $\mathrm{VMR}_{\mathrm{H}_{2} \mathrm{O}}$ since the anomalous $\mathrm{VMR}_{\mathrm{H}_{2} \mathrm{O}}$ scales with its absolute value. The distribution of $s_{\text {anom }}$ of the retrieved dataset is overall shifted towards lower values yielding a negative bias of about $-1.7 \cdot 10^{-4}(37 \%)$ against the reference dataset, which can mostly be attributed to the smoothing error of the retrieval. The smoothing error generally acts by a weakening and thickening of anomalies, which also partly explains the significant positive bias of about $0.8 \mathrm{~km}(28 \%)$ in moisture anomaly thickness $\left(\Delta z_{\text {anom }}\right)$ depicted in Fig. 8(c). Another contributing effect towards the found biases in $s_{\text {anom }}$ and $\Delta z_{\text {anom }}$ is the fact that particularly weak and narrow moisture anomalies are more often completely missed by the retrieval as shown by Fig. 9 .

\subsection{Implications of moisture anomalies for the heating rate profile}

Moisture anomalies affect the heating rate profile by absorbing and emitting infrared radiation. Because of the exponential decrease of water vapor with height, emission at the anomaly top is particularly efficient and can yield a strong local radiative cooling rate (see Fig. 3). We consider this cooling effect to be the moisture anomaly's most prominent footprint on the heating rate profile. In the following, we quantify this cooling effect by considering the minimum heating rate within the vertical bounds of a moisture anomaly, $\min \left(Q_{\mathrm{anom}}\right)$. Since $\min \left(Q_{\mathrm{anom}}\right)$ is a scalar metric, it can intuitively be viewed as a function of moisture anomaly characteristics.

Figure 10(a) and (b) show the joint frequency distributions of the moisture anomaly strength $\left(s_{\text {anom }}\right)$ and $\min \left(Q_{\text {anom }}\right)$ for the tropical ocean dataset and the retrieval dataset, respectively. Both datasets show a clear correlation between the two quantities, namely that stronger anomalies are associated with a stronger peak in radiative cooling. While moisture anomalies with $s_{\text {anom }} \lesssim 10^{-4}$ show similar minimum cooling rates down to about $-2.5 \mathrm{~K} \mathrm{day}^{-1}$ in both the reference and the retrieval dataset, larger differences between the two datasets are apparent for stronger anomalies. The reference dataset (Fig. 10, a) 

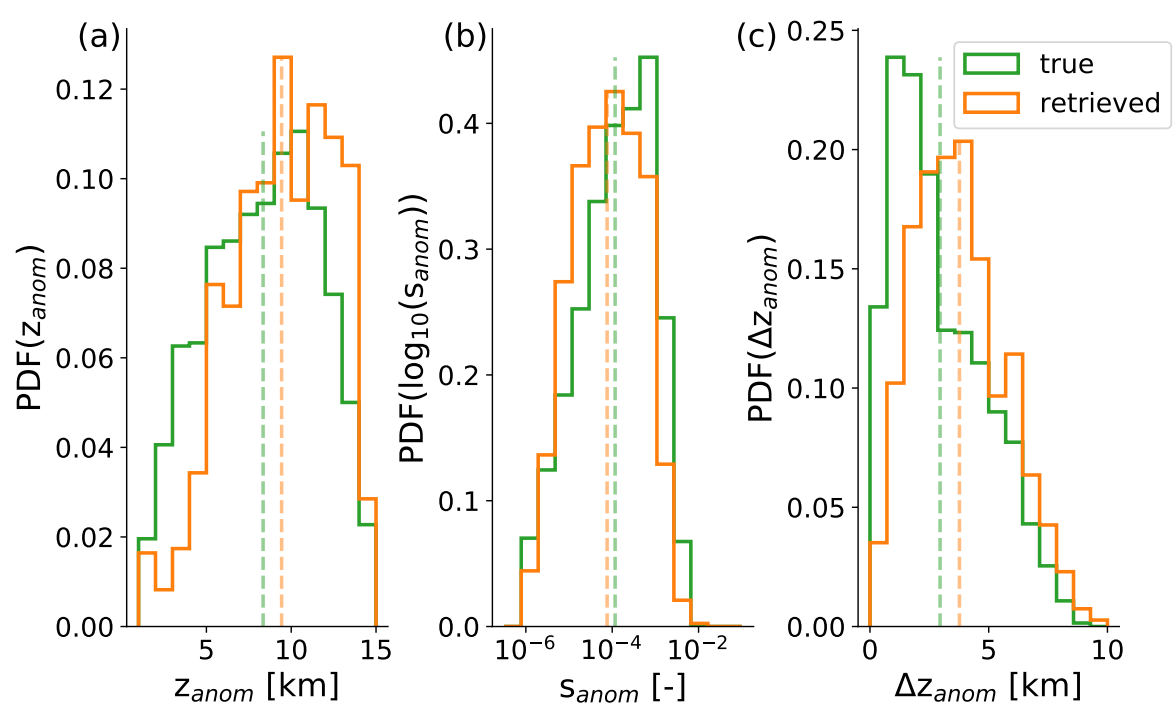

Figure 8. Probability density functions (PDFs) of moisture anomaly characterstics of the tropical ocean reference dataset (denoted as "true") and the retrieved dataset.
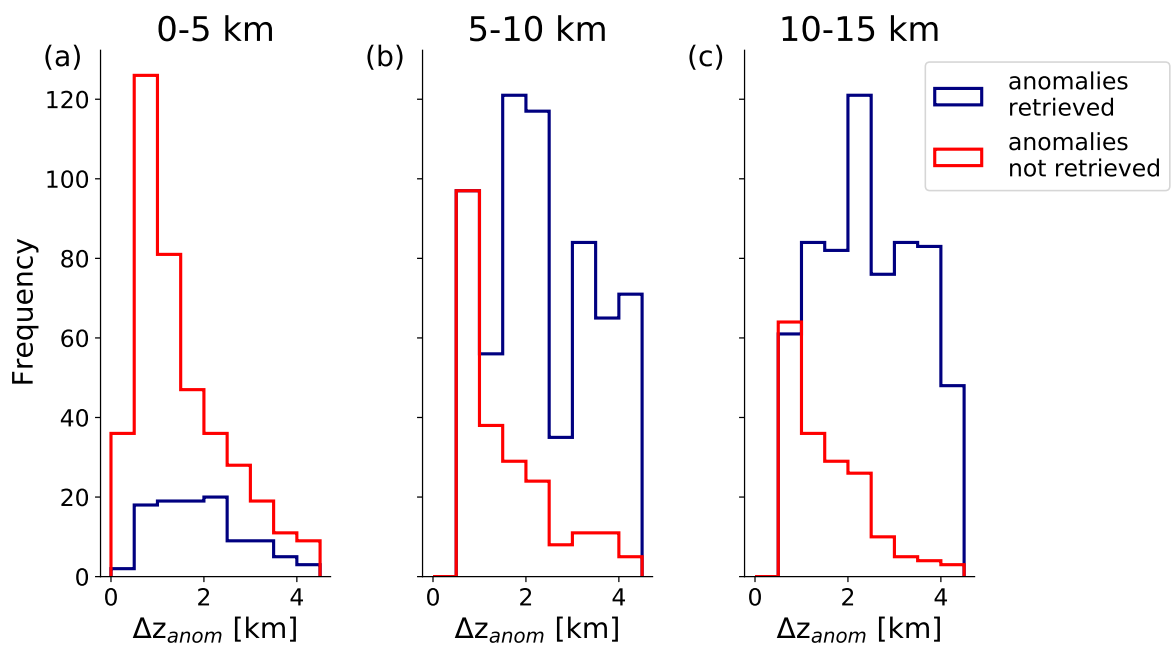

Figure 9. Frequency distributions of moisture anomaly thickness of the tropical ocean reference dataset, split up into cases where a moisture anomaly could be retrieved and could not be retrieved. (a), (b), (c) reflect three altitude regions, namely the lower (0-5 km), mid (5-10 km) and upper $(10-15 \mathrm{~km})$ troposphere.

shows $\min \left(Q_{\text {anom }}\right)$ values between about -1 to $-5 \mathrm{~K}$ day $^{-1}$ for moisture anomalies with $s_{\text {anom }} \gtrsim 10^{-4}$, while the retrieval dataset barely shows any $\min \left(Q_{\text {anom }}\right)$ values smaller than $-3 \mathrm{~K}_{\text {day }}{ }^{-1}$. 
We hypothesise that the increased variability in $\min \left(Q_{\text {anom }}\right)$ for $s_{\text {anom }} \gtrsim 10^{-4}$ in the reference dataset can be attributed to the variability in the exact vertical shapes of the moisture anomalies. Anomalies with a strong negative moisture gradient at their top yield a stronger minimum in radiative cooling, while more smooth anomalies are associated with a less pronounced radiative cooling peak. This effect introduces more variability in $\min \left(Q_{\text {anom }}\right)$ the stronger the anomalies are. It also explains why retrieved moisture anomalies do not show as extreme $\min \left(Q_{\text {anom }}\right)$ values as the reference dataset, since the vertical shape of retrieved anomalies is always bound by the smoothing error.

In the real world, much more extreme vertical moisture gradients associated with moisture anomalies can be observed than in the model based reference dataset used here. Albright et al. (2020) discuss an EML scenario over the Northern Atlantic Trades with a significant moisture drop that is associated with a minimum cooling rate of about $20 \mathrm{~K}$ day $^{-1}$. The results of Fig. 10 indicate that while the retrieval is able to broadly distinguish between differently strong moisture anomalies and their associated heating rates, it is unable to properly represent such extreme cooling rate minima due to smoothing.

Figures $10(\mathrm{c})$ and $(\mathrm{d})$ show the joint frequency distributions of the moisture anomaly strength and height $\left(z_{\text {anom }}\right)$. A clear relation between $s_{\text {anom }}$ and $z_{\text {anom }}$ is found in both datasets, namely that anomalies are weaker the higher up they are in the troposphere. We explain this by the dependence of $s_{\text {anom }}$ on the absolute humidity, which decreases exponentially with height. Combining this with the relation found between anomaly strength and minimum heating rate, it is clear that the radiatively most significant moisture anomalies occur in the lower to mid troposphere. As pointed out in Sect. 6.1 when discussing Fig. 9, the retrieval has particular deficiencies in resolving the rather narrow lower to mid tropospheric moisture anomalies. It is now apparent that this deficiency is particularly relevant, since it affects the strongest and radiatively most significant moisture anomalies. However, the examplary testcase investigated in Sect. 4.1 shows that when the anomaly is relatively strong and the atmosphere aloft has a simple mean structure, also lower to mid tropospheric moisture anomalies can be retrieved. 

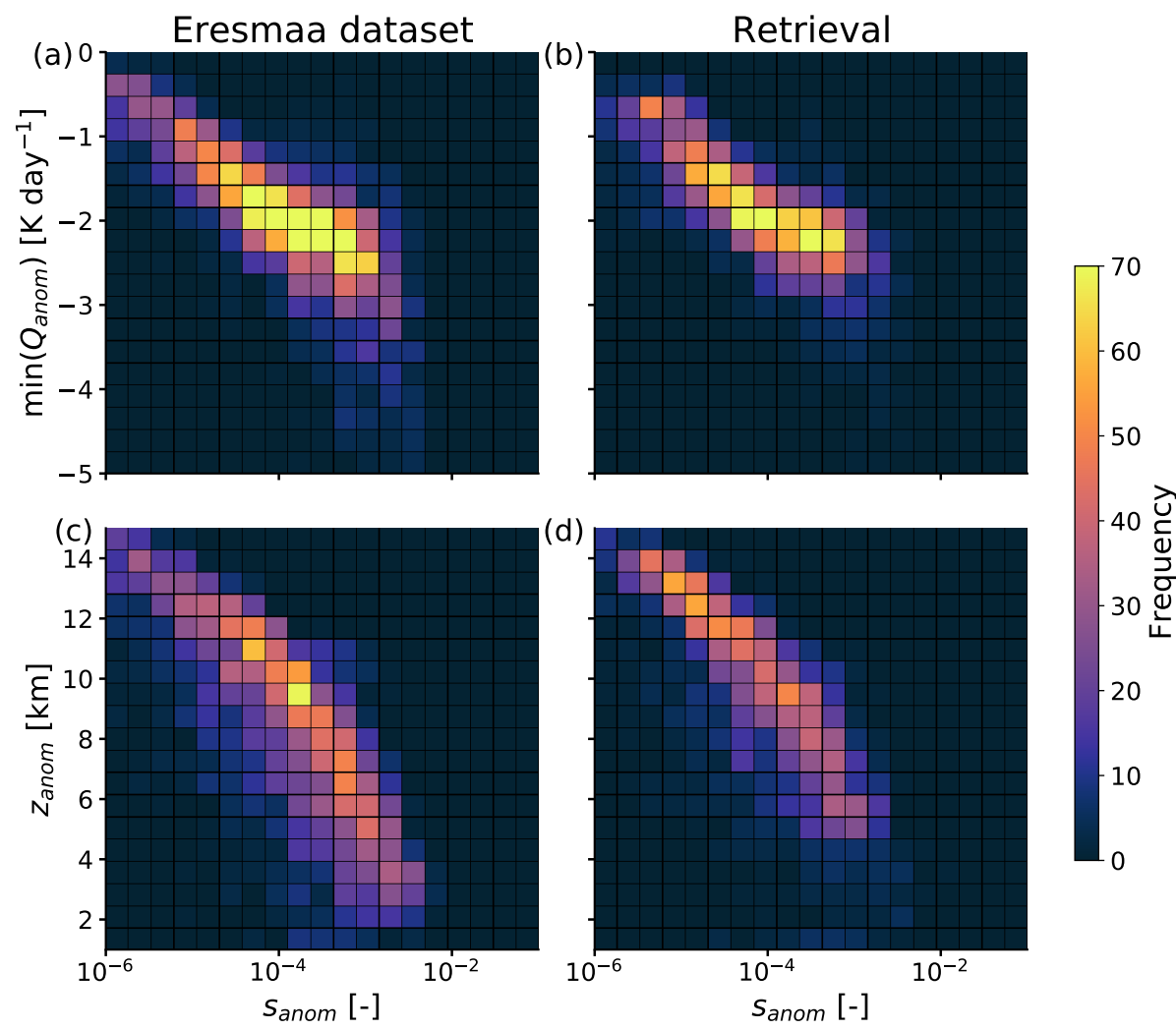

Figure 10. (a) and (b) show the joint frequency distributions of anomaly strength and minimum heating rate within the anomaly layers of the reference dataset and the retrieved dataset, respectively. (c) and (d) show the joint frequency distributions of the anomaly strength and the anomaly height for the two respective datasets.

\section{Summary and conclusions}

The question implicitly raised by the findings of Stevens et al. (2017), whether or not passive satellite retrievals are capable of resolving EMLs, is investigated based on a synthetic retrieval framework where the IASI instrument is represented by the forward model ARTS. An exemplary EML testcase and a set of 1438 tropical ocean model atmospheres are used as input for the forward model and as a reference to evaluate the retrieval results against. The scenes are limited to clear sky.

To make the idea of an EML more graspable and to characterise an EML quantitatively (e.g. by strength, thickness and height), the concept of a moisture anomaly against a loosely fitted but clearly defined reference humidity profile is introduced. Following the ideas of Johnson et al. (1996) and Stevens et al. (2017) about a coupling of EMLs to the freezing level, EMLs would in this framework constitute a subset of rather strong, vertically confined, lower to mid tropospheric positive moisture anomalies. However, for the scope of this work no clear specification of what distinguishes an EML from other moisture anomalies is attempted, which would require a more dedicated selection and analysis of the test dataset. Instead, the clear aim 
of this study is a first systematic evaluation of the retrieval's ability to capture moisture anomalies and their characteristics, with particular focus on the more loosely defined EML cases.

Based on an exemplary testcase similar to the case of Stevens et al. (2017), it is shown that with sufficient independent temperature and water vapor information, a combined retrieval of the moisture and temperature profiles and the surface temperature is capable of resolving the vertical EML structure. The EML signal for the IASI instrument is further characterised by the averaging kernel and the deduced vertical resolution, which is on the order of $1.5 \mathrm{~km}$ for an average tropical ocean atmosphere. However, in the presence of an EML, the strong signal from the EML top weakens the signal from below and introduces a strong gradient in vertical resolution from $0.5 \mathrm{~km}$ at the EML top to $2.5 \mathrm{~km}$ at the EML bottom. This state dependence of vertical resolution motivates a statistical approach to evaluate the retrieval's ability of resolving moisture anomalies in various atmospheric states.

When applying the retrieval to the tropical ocean test dataset, it is found that a large fraction of the absolute retrieval error in humidity can be attributed to smoothing. In particular in the transition region between the boundary layer and the free troposphere, the smoothing error introduces a bias to the retrieved humidity and temperature profiles, which is most likely connected to the sharp humidity drop associated with the stratified barrier between the moist boundary layer and the dry free troposphere in the trade wind region. In the free troposphere, say above $800 \mathrm{hPa}$, the retrieval shows no significant moisture bias, but a positively skewed error variability, indicating that moist anomalies are typically associated with smaller errors than dry anomalies. This is coherent with the idea that dry anomalies that occur beneath moist anomalies are prone to larger errors due to the reduced sensitivity of the satellite measurement below a moist anomaly.

The statistical analysis of the retrieval's performance is completed by a specific evaluation of the retrieval's ability to capture moisture anomalies and their characteristics. It is found that the retrieved moisture anomalies are on average $37 \%$ weaker and $28 \%$ thicker than the anomalies of the reference dataset, which is attributed to smoothing and the fact that rather weak and narrow anomalies are missed by the retrieval more often. While overall about $72 \%$ of moisture anomalies in the reference dataset are identified by the retrieval, a systematic underrepresentation of anomalies below $5 \mathrm{~km}$ is found, where the retrieval only identifies about $30 \%$ of the anomalies present in the reference dataset. This deficiency can mostly be explained by the finding that lower to mid tropospheric moisture anomalies are typically more narrow than anomalies further aloft. Since it is shown that moisture anomalies in the lower to mid troposphere are typically the strongest and radiatively most significant, this issue is quite significant.

The analysis of capturing the moisture anomalies' footprint on the heating rate profiles shows that the retrieval is able to capture the general relation between anomaly strength and minimum cooling rate. However, the retrieval shows a particular shortcoming in capturing the most extreme cooling rates associated with strong lower to mid tropospheric anomalies. We attribute this shortcoming to the retrieval's limited ability of resolving strong vertical moisture gradients that are necessary for the most extreme local cooling rates. Vertical moisture gradients in the real world can be a lot stronger than the ones available from the model test dataset (Albright et al., 2020), which means that retrieval errors with respect to peaks in the cooling rates can be large for rather extreme but realistic cases. 
In summary, the retrieval result of the exemplary EML testcase shows that a passive satellite instrument is in principle capable of resolving a sufficiently strong EML in an otherwise simply structured atmospheric profile. The statistical evaluation of retrieved moisture anomaly characteristics shows that in particular lower to mid tropospheric moisture anomalies are nevertheless frequently missed by the retrieval. While the general dependence of cooling rate extremes on the moisture anomaly strength is captured well by the retrieval, the strongest peaks in radiative cooling associated with strong moisture gradients are not represented well by the retrieval. As a next step we plan to investigate how the presented characterisation of the retrieval's ability to capture moisture anomalies transfers to the application of the retrieval method and analysis tools to real observations. retrieval can be found at https://radiativetransfer.org.

Data availability. The atmospheric profiles over tropical oceans that were used as a basis for the forward modelled IASI spectra and the spectra themselves are publicly available on Zenodo (Prange et al., 2021). The atmospheric profiles are a subset of the ECMWF IFS diverse profile database published by Eresmaa and McNally (2014).

540 Author contributions. MP conducted the radiative transfer and the retrieval calculations and prepared the manuscript. MB and SAB supervised the analysis of the retrieval results, contributed ideas to the manuscript and revised it.

Competing interests. The authors declare that they have no conflict of interest.

Acknowledgements. The authors would like to thank Simon Pfreundschuh, currently at Chalmers University of Technology, for his guidance on the OEM functionality of ARTS. The authors would like to thank Lukas Kluft, currently at the Max Planck Institute for Meteorology in

545 Hamburg, for his guidance on the calculation of heating rates with konrad and for helpful discussions along with Theresa Lang, currently at Universität Hamburg (University of Hamburg). Finally, our thanks go to the ARTS radiative transfer community for their help with using ARTS.

Financial support. This work was funded by the German Research Foundation (DFG) in project 'Elevated Moist Layers - Using HALO during EUREC4A to explore a blind spot in the global satellite observing system', project BU 2253/9-1, part of DFG priority programme HALO SPP 1294, project number 316646266. It also is a contribution to the DFG-funded Cluster of Excellence "CLICCS-Climate, Climatic 
https://doi.org/10.5194/amt-2021-48

Preprint. Discussion started: 6 May 2021

(C) Author(s) 2021. CC BY 4.0 License.

Atmospheric

(c) (1)

Measurement

Techniques

Discussions

Change, and Society" (EXC 2037, Project Number 390683824), and to the Center for Earth System Research and Sustainability (CEN) of Universität Hamburg. 
https://doi.org/10.5194/amt-2021-48

Preprint. Discussion started: 6 May 2021

(C) Author(s) 2021. CC BY 4.0 License.

\section{Atmospheric \\ Measurement \\ Techniques \\ Discussions}

\section{References}

Albright, A. L., Fildier, B., Touzé-Peiffer, L., Pincus, R., Vial, J., and Muller, C.: Atmospheric radiative profiles during EUREC ${ }^{4}$ A, Earth System Science Data, https://doi.org/https://doi.org/10.5194/essd-2020-269, 2020.

Ananthakrishnan, R. and Kesavamurthy, R. N.: Some new features of the vertical distribution of temperature and humidity over Bombay, during the south-west monsoon season, Journal of the Marine Biological Association of India, 14, 732-742, http://mbai.org.in/php/ journaldload.php?id=681\&bkid=45, 1972.

Anderson, G., Clough, S., Kneizys, F., Chetwynd, J., and Shettle, E.: AFGL Atmospheric Constituent Profiles (0.120km), p. 46, 1986.

Bony, S., Stevens, B., Frierson, D. M. W., Jakob, C., Kageyama, M., Pincus, R., Shepherd, T. G., Sherwood, S. C., Siebesma, A. P., Sobel, A. H., Watanabe, M., and Webb, M. J.: Clouds, circulation and climate sensitivity, Nature Geoscience, 8, 261-268, https://doi.org/10.1038/ngeo2398, 2015.

Borger, C., Schneider, M., Ertl, B., Hase, F., García, O. E., Sommer, M., Höpfner, M., Tjemkes, S. A., and Calbet, X.: Evaluation of MUSICA IASI tropospheric water vapour profiles using theoretical error assessments and comparisons to GRUAN Vaisala RS92 measurements, Atmospheric Measurement Techniques, 11, 4981-5006, https://doi.org/https://doi.org/10.5194/amt-11-4981-2018, 2018.

Boukachaba, N., Guidard, V., and Fourrié, N.: Land surface temperature retrieval from IASI for assimilation over the AROME-France domain, 2015.

Buehler, S. A., Mendrok, J., Eriksson, P., Perrin, A., Larsson, R., and Lemke, O.: ARTS, the Atmospheric Radiative Transfer Simulator version 2.2, the planetary toolbox edition, Geoscientific Model Development, 11, 1537-1556, https://doi.org/https://doi.org/10.5194/gmd11-1537-2018, 2018.

Cao, G., Giambelluca, T. W., Stevens, D. E., and Schroeder, T. A.: Inversion Variability in the Hawaiian Trade Wind Regime, Journal of Climate, 20, 1145-1160, https://doi.org/10.1175/JCLI4033.1, 2007.

Clerbaux, C., Boynard, A., Clarisse, L., George, M., Hadji-Lazaro, J., Herbin, H., Hurtmans, D., Pommier, M., Razavi, A., Turquety, S., Wespes, C., and Coheur, P.-F.: Monitoring of atmospheric composition using the thermal infrared IASI/MetOp sounder, Atmospheric Chemistry and Physics, 9, 6041-6054, https://doi.org/https://doi.org/10.5194/acp-9-6041-2009, 2009.

Coppens, D., Meyer, R., Klaes, D., and Montagner, F.: IASI Level 1: Product Guide, https://www-cdn.eumetsat.int/files/2020-04/pdf_iasi_ pg.pdf, 2019.

ECMWF: IFS Documentation - Cy45r1, chap. Part IV : Physical processes, p. 203, ECMWF, https://www.ecmwf.int/node/18714, 2018.

Eresmaa, R. and McNally, A.: Diverse profile datasets from the ECMWF 137-level short-range forecasts, https://doi.org/10.13140/2.1.4476.8963, 2014.

Eriksson, P., Buehler, S., Davis, C., Emde, C., and Lemke, O.: ARTS, the atmospheric radiative transfer simulator, version 2, Journal of Quantitative Spectroscopy and Radiative Transfer, 112, 1551-1558, https://doi.org/10.1016/j.jqsrt.2011.03.001, 2011.

Gordon, I., Rothman, L., Hill, C., Kochanov, R., Tan, Y., Bernath, P., Birk, M., Boudon, V., Campargue, A., Chance, K., Drouin, B., Flaud, J.-M., Gamache, R., Hodges, J., Jacquemart, D., Perevalov, V., Perrin, A., Shine, K., Smith, M.-A., Tennyson, J., Toon, G., Tran, H., Tyuterev, V., Barbe, A., Császár, A., Devi, V., Furtenbacher, T., Harrison, J., Hartmann, J.-M., Jolly, A., Johnson, T., Karman, T., Kleiner, I., Kyuberis, A., Loos, J., Lyulin, O., Massie, S., Mikhailenko, S., Moazzen-Ahmadi, N., Müller, H., Naumenko, O., Nikitin, A., Polyansky, O., Rey, M., Rotger, M., Sharpe, S., Sung, K., Starikova, E., Tashkun, S., Auwera, J. V., Wagner, G., Wilzewski, J., Wcisło, P., Yu, S., and Zak, E.: The HITRAN2016 molecular spectroscopic database, Journal of Quantitative Spectroscopy and Radiative Transfer, 203, 3-69, https://doi.org/https://doi.org/10.1016/j.jqsrt.2017.06.038, 2017. 
https://doi.org/10.5194/amt-2021-48

Preprint. Discussion started: 6 May 2021

(C) Author(s) 2021. CC BY 4.0 License.

Haraguchi, P. Y.: Inversions over the tropical eastern pacific ocean, Monthly Weather Review, 96, 177 - 185, https://doi.org/10.1175/15200493(1968)096<0177:IOTTEP>2.0.CO;2, https://journals.ametsoc.org/view/journals/mwre/96/3/1520-0493_1968_096_0177_iottep_2_ 0_co_2.xml, 1968.

Johnson, R. H., Ciesielski, P. E., and Hart, K. A.: Tropical Inversions near the $0^{\circ} \mathrm{C}$ Level, Journal of the Atmospheric Sciences, 53, 18381855, https://doi.org/10.1175/1520-0469(1996)053<1838:TINTL>2.0.CO;2, 1996.

Johnson, R. H., Rickenbach, T. M., Rutledge, S. A., Ciesielski, P. E., and Schubert, W. H.: Trimodal Characteristics of Tropical Convection, Journal of Climate, 12, 2397-2418, https://doi.org/10.1175/1520-0442(1999)012<2397:TCOTC>2.0.CO;2, https://doi.org/10.1175/ 1520-0442(1999)012<2397:TCOTC>2.0.CO;2, 1999.

Kluft, L. and Dacie, S.: atmtools/konrad: Add line-by-line radiation and conceptual clouds, https://doi.org/http://doi.org/10.5281/zenodo.3899702, 2020.

600 Lacour, J.-L., Risi, C., Clarisse, L., Bony, S., Hurtmans, D., Clerbaux, C., and Coheur, P.-F.: Mid-tropospheric D observations from IASI/MetOp at high spatial and temporal resolution, Atmospheric Chemistry and Physics, 12, 10 817-10 832, https://doi.org/10.5194/acp12-10817-2012, 2012.

Levenberg, K.: A method for the solution of certain non-linear problems in least squares, Quarterly of Applied Mathematics, 2, 164-168, https://doi.org/10.1090/qam/10666, 1944.

Marquardt, D. W.: An Algorithm for Least-Squares Estimation of Nonlinear Parameters, Journal of the Society for Industrial and Applied Mathematics, 11, 431-441, https://doi.org/10.1137/0111030, 1963.

Mauritsen, T. and Stevens, B.: Missing iris effect as a possible cause of muted hydrological change and high climate sensitivity in models, Nature Geoscience, 8, 346-351, https://doi.org/10.1038/NGEO2414, 2015.

Merchant, C. J., Embury, O., Bulgin, C. E., Block, T., Corlett, G. K., Fiedler, E., Good, S. A., Mittaz, J., Rayner, N. A., Berry, D., Eastwood, S., Taylor, M., Tsushima, Y., Waterfall, A., Wilson, R., and Donlon, C.: Satellite-based time-series of sea-surface temperature since 1981 for climate applications, Scientific Data, 6, https://doi.org/https://doi.org/10.1038/s41597-019-0236-x, 2019.

Mlawer, E. J., Taubman, S. J., Brown, P. D., Iacono, M. J., and Clough, S. A.: Radiative transfer for inhomogeneous atmospheres: RRTM, a validated correlated-k model for the longwave, Journal of Geophysical Research: Atmospheres, 102, 16663-16682, https://doi.org/https://doi.org/10.1029/97JD00237, 1997.

615 Mlawer, E. J., Payne, V. H., Moncet, J.-L., Delamere, J. S., Alvarado, M. J., and Tobin, D. C.: Development and recent evaluation of the MT_CKD model of continuum absorption, Philosophical Transactions of the Royal Society A: Mathematical, Physical and Engineering Sciences, 370, 2520-2556, https://doi.org/https://doi.org/10.1098/rsta.2011.0295, 2012.

Muller, C. and Bony, S.: What favors convective aggregation and why?, Geophysical Research Letters, 42, 5626-5634, https://doi.org/10.1002/2015GL064260, 2015.

Posselt, D. J., van den Heever, S. C., and Stephens, G. L.: Trimodal cloudiness and tropical stable layers in simulations of radiative convective equilibrium, Geophysical Research Letters, 35, https://doi.org/https://doi.org/10.1029/2007GL033029, 2008.

Prange, M., Brath, M., and Buehler, S. A.: Supplementary data for "Are elevated moist layers a blind spot for hyperspectral infrared sounders? - a model study", https://doi.org/10.5281/zenodo.4501184, 2021.

Rodgers, C. D.: Inverse Methods for Atmospheric Sounding, https://doi.org/https://doi.org/10.1142/3171, 2000.

625 Romps, D. M.: An Analytical Model for Tropical Relative Humidity, Journal of Climate, 27, 7432-7449, https://doi.org/https://doi.org/10.1175/JCLI-D-14-00255.1, 2014. 
https://doi.org/10.5194/amt-2021-48

Preprint. Discussion started: 6 May 2021

(c) Author(s) 2021. CC BY 4.0 License.

(c) (1)

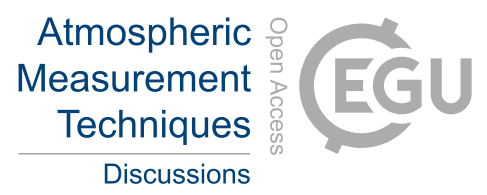

Schneider, M. and Hase, F.: Optimal estimation of tropospheric $\mathrm{H} 2 \mathrm{O}$ and \&deltaD with IASI/METOP, Atmospheric Chemistry and Physics, 11, 11 207-11 220, https://doi.org/10.5194/acp-11-11207-2011, 2011.

Sobel, A. H. and Bretherton, C. S.: Modeling Tropical Precipitation in a Single Column, Journal of Climate, 13, 4378-4392, https://doi.org/10.1175/1520-0442(2000)013<4378:MTPIAS>2.0.CO;2, https://doi.org/10.1175/1520-0442(2000)013<4378:MTPIAS> 2.0.CO;2, 2000.

Stevens, B., Brogniez, H., Kiemle, C., Lacour, J.-L., Crevoisier, C., and Kiliani, J.: Structure and Dynamical Influence of Water Vapor in the Lower Tropical Troposphere, Surveys in Geophysics, 38, 1371-1397, https://doi.org/https://doi.org/10.1007/s10712-017-9420-8, 2017.

Zuidema, P.: The 600-800-mb Minimum in Tropical Cloudiness Observed during TOGA COARE, Journal of the Atmospheric Sciences, 55, 2220-2228, https://doi.org/10.1175/1520-0469(1998)055<2220:TMMITC>2.0.CO;2, https://doi.org/10.1175/ 1520-0469(1998)055<2220:TMMITC>2.0.CO;2, 1998. 